\title{
The Role of Moisture in the Dynamics and Energetics of Turbulent Baroclinic Eddies
}

\author{
G. LAPEYRE \\ Atmospheric and Oceanic Sciences Program, Princeton University, Princeton, New Jersey \\ I. M. HELD \\ NOAA/Geophysical Fluid Dynamics Laboratory, Princeton, New Jersey
}

(Manuscript received 15 September 2003, in final form 20 January 2004)

\begin{abstract}
The effects of moisture on nonlinear baroclinic eddies are examined in the context of a horizontally homogeneous two-layer quasigeostrophic model. Using an explicit equation for moisture and a simple parameterization of latent heat release, the present study focuses on how moisture affects the statistically steady state of a baroclinically unstable flow. It is shown that, when latent heating is weak, the flow is dominated by jets and baroclinic waves, just as in the corresponding dry model. In this regime, the concept of an effective static stability can be used, which allows one to interpret some aspects of the moist simulations in terms of an equivalent dry model. It is found that a useful way of diagnosing the effective static stability is by relating it to the eddy fluxes of moisture and moist potential vorticity; no a priori theory for its value is presented here. As the strength of latent heating is increased, the flow rather suddenly becomes vortex dominated, with an asymmetry between strong low-level cyclones and weak anticyclones that has no analog in the dry model. It is argued that this asymmetry develops because of a correlation between low-level vorticity and moisture that results from the correlated horizontal transports of moisture and vorticity. The energetics of the simulations and the efficiency of energy production by latent heat release are discussed.
\end{abstract}

\section{Introduction}

Studies of the effect of moisture on baroclinic eddies have focused primarily on initial value problems. One body of work isolates the linear growth phase using quasigeostrophic (Bannon 1986; Mak 1982; Wang and Barcilon 1986), semigeostrophic (Emanuel et al. 1987; Joly and Thorpe 1991; Montgomery and Farrell 1991), or primitive (Fantini 1990; Whitaker and Davis 1994) equations. Others study the eddy life cycle starting from linear growth on zonally symmetric flows with the primitive equations (Balasubramanian and Garner 1997; Balasubramanian and Yau 1996; Gall 1976; Gutowski et al. 1992) or with the quasigeostrophic theory (Beare et al. 2003). Yet another set of studies, in a weather prediction context, examines the importance of latent heat release for particular events (Davis 1992; Gyakum 1983; Zhang and Harvey 1995). There is relatively little discussion of how the presence of latent heat release affects the statistically steady state of a baroclinically unstable system. Full general circulation model simulations in which latent heat release is removed or weakened, or in which the mean temperature is greatly altered, are

Corresponding author address: G. Lapeyre, LPO, IFREMER, BP 70, 29280 Plouzané, France.

E-mail: glapeyre@ifremer.fr informative in this regard, but, in addition to other complexities, dramatic changes in the Tropics can confound attempts at isolating the in situ effects of latent heat release in midlatitudes (Boer 1995; Hall et al. 1994; Hayashi and Golder 1981; Stephenson and Held 1993; Zhang 1995). More idealized models of moist midlatitude storm tracks are needed to address these issues. An understanding of moist storm tracks is clearly relevant for studying the responses of midlatitudes to global warming (Held 1993).

We choose a quasigeostrophic (QG) two-layer model in this work to examine some of the fundamental properties of the statistically steady moist baroclinic system. The quantitative limitations of QG dynamics for studies of midlatitude dynamics are well known, and can be particularly evident when examining the effects of moisture on linear baroclinic growth (e.g., Emanuel et al. 1987), but the hope is that the simplicity of the QG framework will lead to new qualitative insights. One of the problems in using QG moist models is that one assumes in QG that horizontal structure in the static stability is small. However, the effective static stability is reduced in regions of latent heat release, potentially creating large changes in the horizontal in this effective stability. The reader should keep this limitation in mind as we try to take advantage of the simplicity of QG 
dynamics to isolate concepts that might have more general validity.

In constructing our two-layer model, we closely follow Zhang (1995) but we have chosen to simplify the problem even further by considering a horizontally homogeneous, doubly periodic geometry. The close relationship between horizontal homogeneous turbulence models and more traditional inhomogeneous models has been demonstrated by Pavan and Held (1996) in the dry two-level QG model. This close correspondence implies that one can use the homogeneous framework to test closure theories for eddy heat fluxes in the cleanest possible setting (Held and Larichev 1996). Eddy vorticity and momentum fluxes vanish identically in these homogeneous simulations, and eddy potential vorticity fluxes are identical to eddy heat, or thickness, fluxes to within a constant of proportionality. Most importantly, one need not, as in inhomogeneous models, simultaneously consider the effects of baroclinic eddies on the larger-scale environment and the effects of this environment on the baroclinic eddies; rather one can focus exclusively on the latter. These simplifications justify the abstraction of this homogeneous setting, in our view.

In section 2, we introduce our QG model of moist baroclinic turbulence and discuss briefly what range of parameters seems most relevant for the atmosphere. In section 3, limiting cases are presented to introduce the basic dynamics of the moist model. In section 4, we examine the statistically steady states when we vary some of the parameters controlling the moist dynamics. Sections 5 and 6 are devoted to a discussion of the underlying processes that control moist turbulence in this model while section 7 contains our conclusions.

\section{Description of the model}

\section{a. Model equations}

The two-layer quasigeostrophic homogeneous model on the $\beta$ plane with bottom Ekman friction is used as the starting point for our moist model. The dimensional vorticity equations are

$$
\begin{aligned}
& \frac{D_{1}}{D t}\left(\nabla^{2} \psi_{1}+\beta y\right)+f_{0} \boldsymbol{\nabla} \cdot \mathbf{u}_{1}=F_{1}, \\
& \frac{D_{2}}{D t}\left(\nabla^{2} \psi_{2}+\beta y\right)+f_{0} \boldsymbol{\nabla} \cdot \mathbf{u}_{2}=F_{2}-r \nabla^{2} \psi_{2} .
\end{aligned}
$$

Subscripts 1 and 2 refer to upper and lower layers. In the quasigeostrophic theory, $D_{i} / D t$ is the horizontal advection by the nondivergent (geostrophic) velocity field $\mathbf{v}_{i}=\left(-\partial_{y} \psi_{i},+\partial_{x} \psi_{i}\right)$, whereas $\boldsymbol{\nabla} \cdot \mathbf{u}_{i}$ corresponds to the divergence of ageostrophic motions (see Holton 1972, chapter 6). Terms $F_{1}$ and $F_{2}$ correspond to small-scale dissipation, the formulation for which is described in the appendix.

Mass conservation in each layer takes the QG form

$$
\frac{D_{i} h_{i}}{D t}+H_{i} \boldsymbol{\nabla} \cdot \mathbf{u}_{i}=-(-1)^{i} S
$$

where $H_{i}$ is the mean depth of layer $i$ and $h_{i}$ is the departure from this mean. The upper and lower surfaces are assumed to be flat and rigid, with only the interface between the layers changing in time, so that $h_{1}+h_{2}=0$. We use the notation $\eta \equiv h_{1}=-h_{2}$ for the interface displacement. We also choose $H_{1}=H_{2} \equiv H_{0}$, but retain this more general notation for later reference; $S$ accounts for the mass exchange from the lower to the upper layer:

$$
S \equiv \frac{(L P-R) H_{2}}{c_{p} \delta \Theta},
$$

where $L$ is the latent heat of vaporization, $c_{p}$ the heat capacity, $P$ the precipitation per unit of mass of the lower layer, while $R$ is the radiative cooling rate, and $\delta \Theta$ the difference in potential temperature between the two layers.

We relate the baroclinic streamfunction to the interface height in the usual way, $f_{0}\left(\psi_{1}-\psi_{2}\right)=g^{*} \eta$, with $g^{*} \equiv g \delta \Theta / \Theta_{0}$. The dry potential vorticity (PV) in each layer is

$$
\begin{aligned}
q_{i} & =\nabla^{2} \psi_{i}-f_{0} \frac{h_{i}}{H_{i}}+\beta y \\
& =\nabla^{2} \psi_{i}+(-1)^{i} \lambda_{i}^{-2}\left(\psi_{1}-\psi_{2}\right)+\beta y,
\end{aligned}
$$

where $\lambda_{i} \equiv \sqrt{g^{*} H_{i}} / f_{0}$ is the Rossby deformation radius in each layer. The evolution of PV is governed by

$$
\begin{aligned}
& \frac{D_{1} q_{1}}{D t}=-\frac{f_{0} S}{H_{1}}+F_{1}, \\
& \frac{D_{2} q_{2}}{D t}=+\frac{f_{0} S}{H_{2}}+F_{2}-r \nabla^{2} \psi_{2} .
\end{aligned}
$$

Consistent with the treatment of moisture as a thermodynamic variable analogous to temperature, or layer thickness, we assume that the mixing ratio departs from its reference value $m_{0}$ by small amounts $m$, so that

$$
\frac{D_{2} m}{D t}+m_{0} \boldsymbol{\nabla} \cdot \mathbf{u}_{2}=E-P+F_{m},
$$

where $E$ is the evaporation rate per unit mass of the lower layer, and $F_{m}$ represents small-scale dissipation. We believe this linearization of the ageostrophic advection is essential to maintain a consistent QG framework. Here, moisture is assumed to exist only in the lower layer to reflect the vertical stratification of humidity in the atmosphere. We determine precipitation by simply resetting $m$ to equal $m_{s}$, the saturation value, whenever $m$ exceeds $m_{s}$.

We allow the saturation moisture to increase linearly with increasing "temperature," or interface displacement,

$$
\frac{m_{s}}{m_{0}}=C \frac{\eta}{H_{2}}=\mathcal{C} \frac{f_{0}}{g^{*} H_{2}}\left(\psi_{1}-\psi_{2}\right),
$$


where $C$ is nondimensional. Recall that both $m$ and $m_{s}$ are departures from $m_{0}$ and can take on either sign. Since the relative perturbations in mixing ratio are assumed to be small, it is natural to simply linearize the ClausiusClapeyron equation in this manner.

By eliminating the precipitation from the interface and moisture equations, we obtain a moist static energylike variable, $\eta / H_{2}+\mathcal{L} \mathrm{m} / \mathrm{m}_{0}$, which satisfies

$\frac{D_{2}}{D t}\left(\frac{\eta}{H_{2}}+\frac{\mathcal{L} m}{m_{0}}\right)=(1-\mathcal{L}) \boldsymbol{\nabla} \cdot \mathbf{u}_{2}+\frac{\mathcal{L}(E-R / L)}{m_{0}}$,

where we have omitted the small-scale dissipation for convenience. The parameter $\mathcal{L}$ is a nondimensional measure of the strength of latent heating:

$$
\mathcal{L} \equiv \frac{L m_{0}}{c_{p} \delta \Theta} .
$$

We can then form a moist PV in the lower layer,

$$
q_{m}=\nabla^{2} \psi_{2}+\frac{1}{1-\mathcal{L}}\left[\lambda_{2}^{-2}\left(\psi_{1}-\psi_{2}\right)+f_{0} \mathcal{L} \frac{m}{m_{0}}\right]+\beta y,
$$

which satisfies

$$
\frac{D_{2} q_{m}}{D t}=\frac{\mathcal{L}}{1-\mathcal{L}} \frac{f_{0}(E-R / L)}{m_{0}}-r \nabla^{2} \psi_{2},
$$

where we have omitted the small-scale dissipation once again. This two-layer model is distinctive in that it possesses a moist PV, in the lower layer, that is exactly conserved in the absence of dissipation, evaporation, and radiative heating. This is not generally the case in a continuously stratified flow, as a term proportional to $J\left(\partial_{z} \psi, m\right)$ corresponding to $\nabla \eta \times \nabla m$ would appear on the rhs of (4) (see Cao and Cho 1995 for a derivation). Therefore, even in this two-layer model, this simple conservation law for moist PV is modified if we include moisture in the upper as well as lower layers.

This is effectively the model of Zhang (1995). We now take the additional step of creating a horizontally homogeneous model. As in the studies of Salmon (1978), Haidvogel and Held (1980), and Held and Larichev (1996), we begin by specifying a uniform zonal flow in each layer $U_{i}$, or, equivalently, a uniform interface slope $\partial \bar{\eta} / \partial y=-f_{0}\left(U_{1}-U_{2}\right) / g *$, and uniform environmental PV gradients in each layer $\beta+$ $(-1)^{i+1} U \lambda_{i}^{-2}$, where $U \equiv U_{1}-U_{2}$. We then assume that the departures from these mean values of PV or streamfunction are doubly periodic in a square domain.

To maintain homogeneity in the moist model, we require first of all that the evaporation $E$ and the radiative cooling $R$ be horizontally homogeneous; we simply set both to spatially uniform constants. In fact, we need to set them so that $\mathrm{LE}=R$ to obtain a steady state. This has the result that the domain-averaged moist static energy and moist PV are independent of time. In the presence of a thickness gradient, it is also natural to assume the existence of an environmental moisture gradient. To ensure homogeneity we require that this moisture gradient be the gradient of the saturated moisture, consistent with the imposed temperature gradient; that is,

$$
m=m_{0} C \frac{\bar{\eta}(y)}{H_{2}}+\langle m\rangle+m^{\prime} .
$$

Here, $\langle m\rangle$ is the global mean of $m, m^{\prime}$ is doubly periodic, and $\bar{\eta}(y)=-f_{0} U y / g *$ is the large-scale interface displacement with $y=0$ in the center of the domain. If we did not constrain the moisture gradient in this way, then the degree of saturation of the flow would vary across the domain. We choose not to examine such a situation.

It remains to predict $\langle m\rangle$. We would like the evaporation $E$ to serve as a parameter that allows one to force the system to be more or less saturated. We proceed by retaining equations for the domain-averaged moisture $\langle m\rangle$ and interface displacement $\langle\eta\rangle$ :

$$
\begin{aligned}
\frac{\partial}{\partial t}\left(\frac{\langle m\rangle}{m_{0}}\right) & =-\frac{P-E}{m_{0}}, \\
\frac{\partial}{\partial t}\left(\frac{\langle\eta\rangle}{H_{2}}\right) & =\frac{\mathcal{L}(P-E)}{m_{0}} .
\end{aligned}
$$

Since the domain-averaged moist static energy $\left\langle\eta / H_{2}+\right.$ $\left.\mathcal{L} \mathrm{m} / \mathrm{m}_{0}\right\rangle$ is conserved, we assume for simplicity that it is zero; $\langle m\rangle$ and $\langle\eta\rangle$ are then proportional, and we need only retain an equation for the mean moisture. The latter is driven by the difference between the specified evaporation and the predicted domain average of the precipitation. Precipitation is defined so as to prevent $(\langle m\rangle$ $\left.+m^{\prime}\right) / m_{0}$ from rising above $C\left(\langle\eta\rangle+\eta^{\prime}\right) / H_{2}$, where the prime denotes the deviation from the domain mean. Equivalently, $(1+C \mathcal{L})\langle m\rangle / m_{0}+m^{\prime} / m_{0}$ is prevented from rising above $\mathrm{C} \eta^{\prime} / \mathrm{H}_{2}$.

We nondimensionalize all the variables by choosing $\lambda=\lambda_{1}=\lambda_{2}$ as the length scale, $\lambda / U$ as the time scale, $m_{0} U /\left(f_{0} \lambda\right)$ as the scale for the mixing ratio, and therefore $m_{0} U^{2} /\left(f_{0} \lambda^{2}\right)$ as the scale for precipitation and evaporation. We choose $U_{1}=-U_{2}=U / 2$. Omitting the subgrid mixing terms for clarity, our model equations take the following form, in which all dynamical quantities now refer to deviations from the domain mean unless explicitly indicated (in particular, precipitation $=$ $\Pi+P$, where $\Pi$ is the domain mean and $P$ the deviation from this mean, while $\mathcal{M}$ is the mean moisture):

$$
\begin{aligned}
\frac{D_{1} q_{1}}{D t}= & -\frac{1}{2} \frac{\partial}{\partial x} q_{1}-(\mathcal{B}+1) \frac{\partial}{\partial x} \psi_{1}-\mathcal{L} P, \\
\frac{D_{2} q_{2}}{D t}= & +\frac{1}{2} \frac{\partial}{\partial x} q_{2}-(\mathcal{B}-1) \frac{\partial}{\partial x} \psi_{2}+\mathcal{L} P \\
& -\mathcal{R} \nabla^{2} \psi_{2}, \\
\frac{D_{2} m}{D t}= & +\frac{1}{2} \frac{\partial}{\partial x} m+\mathcal{C} \frac{\partial}{\partial x} \psi_{2}-P-\nabla \cdot \mathbf{u}_{2},
\end{aligned}
$$




$$
\frac{\partial}{\partial t} \mathcal{M}=\mathcal{E}-\Pi,
$$

where precipitation $\Pi+P$ resets $(1+C \mathcal{L}) \mathcal{M}+m$ equal to $C\left(\psi_{1}-\psi_{2}\right)$ whenever it rises above this value. Also,

$$
\begin{aligned}
q_{i} & =\nabla^{2} \psi_{i}+(-1)^{i}\left(\psi_{1}-\psi_{2}\right), \\
\frac{D_{i}}{D t} & =\frac{\partial}{\partial t}+J\left(\psi_{i},\right),
\end{aligned}
$$

with $i=1$ or 2 . The nondimensional parameters are

$$
\mathcal{B} \equiv \frac{\beta \lambda^{2}}{U}, \quad \mathcal{R}=\frac{r \lambda}{U}, \quad \mathcal{E}=\frac{E f_{0} \lambda^{2}}{m_{0} U^{2}},
$$

and also $\mathcal{L}$ defined by (3). Here, $\mathcal{E}$ is the ratio of the evaporation per unit of mass of the lower layer to the vertical advection by the eddies of the mean moisture $m_{0} \boldsymbol{\nabla} \cdot \mathbf{u}_{2}$, the latter scaling as $m_{0} U^{2} / f_{0} \lambda^{2}$.

Among the derived variables of interest, the moist PV equation in the lower layer is

$$
\frac{D_{2}}{D t} q_{m}=+\frac{1}{2} \frac{\partial}{\partial x} q_{m}-\Gamma \frac{\partial}{\partial x} \psi_{2},-\mathcal{R} \nabla^{2} \psi_{2}
$$

where

$$
q_{m}=\nabla^{2} \psi_{2}+\frac{1}{1-\mathcal{L}}\left(\psi_{1}-\psi_{2}+\mathcal{L} m\right)
$$

and $\Gamma$ is the imposed meridional gradient of the moist $\mathrm{PV}$ :

$$
\Gamma \equiv \mathcal{B}-\frac{1+\mathcal{C L}}{1-\mathcal{L}}
$$

[the small-scale dissipation terms have once again been omitted in (6)].

One can integrate the moist and dry PV equations [Eqs. (5a), (5b), and (6)] forward in time and then obtain the mixing ratio from the difference, so as to avoid computing the divergence explicitly, or one can obtain the divergence diagnostically from the vorticity and thickness equations, and integrate the moisture equation (5c) directly. We have used this second method. The numerical model integrates Eq. (5a)-(5d) in a doubly periodic domain using a pseudospectral code with a leapfrog adaptive time step, adapted from Smith et al. (2002) and some of its specificities are explained in the appendix. The horizontal resolution is $256 \times 256$ grid points. The model precipitation would likely be better behaved treating water vapor as a grid rather than spectral variable and advecting it with a monotonic scheme, but we leave this possible improvement for future work. A small number of runs were performed with resolution up to $1024 \times 1024$. The added resolution results in more aesthetically pleasing precipitation distributions, but our tentative conclusion is that the large-scale structure and energetics of the flow are insensitive to these details.

\section{b. Parameter selection}

Even if it is the case that the subgrid-scale diffusion, resolution, and the size of the domain are not significant to the results, we still have five parameters $(\mathcal{B}, \mathcal{R}, \mathcal{L}, \mathcal{C}$, $\mathcal{E})$, resulting in a large parameter space of which we can only explore small sections. For our main series of experiments we fix the dry parameters and focus on certain variations in two of the three moist parameters.

The equal-depth dry homogeneous two-layer model is characterized by two nondimensional parameters: $\mathcal{B}$ a nondimensional $\beta$ or supercriticality $\chi \equiv \mathcal{B}^{-1}$ (the inviscid dry model is unstable when $\chi>1$ ), and a nondimensional surface drag coefficient $\mathcal{R}$. The dry parameters were chosen to represent a modestly supercritical state for baroclinic instability, with $\mathcal{B}=0.78$. Starting with relatively weak instability in the dry case allows the eddy energy to grow substantially in the moist case while retaining a computationally efficient model with a reasonable time step. For the earth's atmosphere, one can estimate $\mathcal{B}^{-1}$ as $I H / a$ where $I$ is the isentropic slope, $H$ the typical height of the troposphere and $a$ the earth radius. Its typical value is observed around 1 . The Ekman dissipation at the boundary layer gives an $e$ folding time of the order $r^{-1} \approx 4$ days. We choose a frictional parameter, moderately strong in accordance to this value; that is, $R=0.16$.

The periodic domain is a square of size $18 \pi \lambda$, corresponding to a smallest nonzero wavenumber of $1 /(9 \lambda)$ that allows some resolution of the cascades of PV and moisture to small scales while leaving room between the energy containing eddies and the size of the domain. In the following, we will call the simulation with $\mathcal{L}=$ 0 the passive case.

Regarding moist parameters, we first note that $C$ can be thought of as the absolute value of the ratio of the slope of isolines of the mean mixing ratio to the mean isentropic slope. Assuming that the mixing ratio distribution is dominated by that of temperature, then $C$ is roughly the ratio of the slope of the mean temperature isolines to the isentropic slope. Since the horizontal gradients of temperature and potential temperature are the same, and given that the vertical gradient of potential temperature is roughly a factor of 2 smaller than that of temperature $\left(3 \mathrm{~K} \mathrm{~km}^{-1}\right.$ as opposed to $\left.6.5 \mathrm{~K} \mathrm{~km}^{-1}\right)$, we find that $C \approx 2$.

There are several alternatives for estimating $\mathcal{L}$. On the one hand, it has been argued that a model of the storm track should always produce convection, or $\mathcal{L} \approx$ 1 , in the warm sector of surface cyclones (Emanuel 1985; Juckes 2000). But our QG model is constrained to have a uniform value of $\mathcal{L}$. A much smaller value for the domain average of $\mathcal{L}$ can be justified if we take $\delta \Theta_{0}$ $\approx 15 \mathrm{~K}$ and estimate $m_{0}$ from the mixing ratio averaged over the lower half of the extratropical troposphere (from Peixoto and Oort 1991). We then find $\mathcal{L} \approx 0.2$ in $\mathrm{NH}$ winter and 0.35 in $\mathrm{NH}$ summer. The product $C \mathcal{L}$ controls the amount of latent heat release as a parcel 
cools while remaining saturated. The resulting values for the winter and summer are $C \mathcal{L} \approx 0.4$ and 0.7 , respectively.

If one chooses $U \approx 20 \mathrm{~m} \mathrm{~s}^{-1}, \lambda \approx 1000 \mathrm{~km}$, and an evaporation time scale $m_{0} / E \approx 7.5$ days, one estimates $\mathcal{E} \approx 0.4$. We have used the larger value of $\mathcal{E}=1.39$ for most of our simulations. This value results in saturation through most of the domain in the passive limit. This choice allows us to study the desaturation of the air with increasing latent heating strength, thereby testing more thoroughly our ideas concerning the best way to define an effective static stability. It still results in saturation in a small fraction of the domain for plausible values of $\mathcal{L}$.

We cannot expect dry and moist models in this homogeneous framework to both closely mimic the atmosphere, holding other parameters fixed, especially given the large differences in eddy energy that emerge. But this model allows us to focus on particular dynamical processes more difficult to identify in a more complex framework.

\section{Limiting cases}

In thinking about the effects of moisture, it is useful to keep in mind two limiting cases: the passive limit $(\mathcal{L} \rightarrow 0)$ and the saturated limit $(\mathcal{E} \rightarrow \infty)$.

\section{a. Passive moisture transport}

Eddy moisture transport can be divided into two parts: the flux of saturated moisture, and the flux of the moisture deficit $m-m_{s}<0$ :

$$
\langle v m\rangle=\left\langle v m_{s}\right\rangle+\left\langle v\left(m-m_{s}\right)\right\rangle .
$$

The saturated flux is known once the heat flux is known so its dynamics can be understood from the dynamics of temperature. On the other hand, the moisture deficit flux has a different relation to the dynamics and is the new feature of this moist model. We shall show that the beta effect exerts a surprisingly strong control on the flux of moisture deficit in this model. This control is most easily understood in the passive case. Indeed, it is helpful to simplify the equations even further as a first step, by removing the effects of precipitation from the moisture equation, leaving this variable as a passive tracer advected by both the geostrophic and ageostrophic flow. A linear analysis of growing baroclinic waves serves to illustrate the relation. In this linear analysis, we also ignore both Ekman friction and small-scale dissipation.

Using the notation

$$
d_{2} \equiv \frac{\partial}{\partial t}+U_{2} \frac{\partial}{\partial x}=\frac{\partial}{\partial t}-\frac{1}{2} \frac{\partial}{\partial x},
$$

we have the following equations for $m, \eta \equiv \psi_{1}-\psi_{2}$, $m-m_{s} \equiv m-c \eta$, $\zeta_{2}$, and $q_{2}=\zeta_{2}+\eta$ :

$$
\begin{aligned}
d_{2} m & =-\boldsymbol{\nabla} \cdot \mathbf{u}_{2}+c \frac{\partial}{\partial x} \psi_{2}, \\
d_{2} \eta & =+\boldsymbol{\nabla} \cdot \mathbf{u}_{2}+\frac{\partial}{\partial x} \psi_{2}, \\
d_{2}\left(m-m_{s}\right) & =-(1+\mathcal{C}) \boldsymbol{\nabla} \cdot \mathbf{u}_{2}, \\
d_{2} \zeta_{2} & =-\boldsymbol{\nabla} \cdot \mathbf{u}_{2}-\mathcal{B} \frac{\partial}{\partial x} \psi_{2}, \quad \text { and } \\
d_{2} q_{2} & =-(\mathcal{B}-1) \frac{\partial}{\partial x} \psi_{2} .
\end{aligned}
$$

Note that there is no meridional gradient of moisture deficit in this model. We can combine the moisture deficit and vorticity equations to eliminate the divergence:

$$
d_{2}\left[m-m_{s}-(1+c) \zeta_{2}\right]=\mathcal{B}(1+c) \frac{\partial}{\partial x} \psi_{2} .
$$

In the special case that $\mathcal{B}=0$, the rhs vanishes, and, if we consider an exponentially growing normal mode, the quantity $m-m_{s}-(1+c) \zeta_{2}$ must vanish as well. But the domain average of the meridional vorticity flux is constrained to be identically zero. Therefore, $\left\langle v_{2}\left(m-m_{s}\right)\right\rangle=$ 0 as well. More generally, with $\mathcal{B} \neq 0$, we can combine with the $q_{2}$ equation to obtain $d_{2} \xi=0$, where

$$
\xi \equiv m-m_{s}-(1+\mathcal{C}) \zeta_{2}+\frac{\mathcal{B}(1+\mathcal{C})}{\mathcal{B}-1} q_{2} .
$$

For an unstable normal mode, we will have $\xi=0$, so the flux of moisture deficit is proportional to the flux of PV in the lower layer:

$$
\left\langle v_{2}\left(m-m_{s}\right)\right\rangle=\frac{\mathcal{B}(1+\mathcal{C})}{1-\mathcal{B}}\left\langle v_{2} q_{2}\right\rangle
$$

(there is no contribution from the flux of vorticity). Since $\mathcal{B}-1$ is the gradient of PV in the lower layer, we can rewrite this expression in terms of the diffusivity for $\mathrm{PV}$ in the lower layer $\mathcal{D}_{2}=-\left\langle v_{2} q_{2}\right\rangle /(\mathcal{B}-1)$ :

$$
\left\langle v_{2}\left(m-m_{s}\right)\right\rangle=\mathcal{B}(1+C) \mathcal{D}_{2} .
$$

This result is exact for a growing normal mode, but it continues to hold with considerable accuracy for the fully turbulent nonlinear model. We generate a statistically steady state of the dry model, in the presence of Ekman damping and small-scale dissipation, for several values of supercriticality, and with $C=0$ for simplicity, and advect a passive moisture variable with this flow. We continue to set precipitation equal to zero, so the variance in this passive tracer is generated by advection across the mean horizontal and vertical gradients (in this case with $\mathcal{C}=0$ there is only a vertical gradient). The resulting flux of $m-m_{s}$ is plotted against the PV diffusivity and compared against the prediction $\left\langle v_{2}\left(m-m_{s}\right)\right\rangle=\mathcal{B D}_{2}$ in Fig. 1a. We see clearly that the nonlinear model reproduces correctly the same feature as the growing normal mode. The result (9) is found to 
(a)

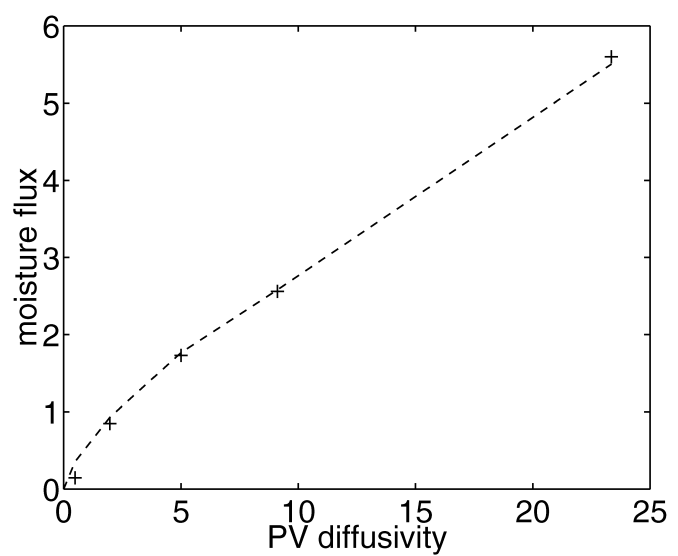

(b)

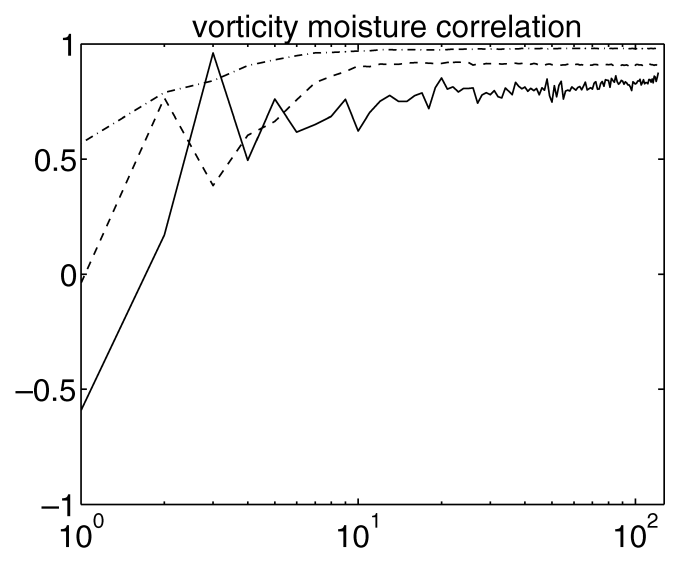

FIG. 1. (a) Moisture flux plotted as a function of PV diffusivity in turbulent simulations with no precipitation, for different values of $\mathcal{B}$. The dashed curve corresponds to the prediction (9). (b) Correlation coefficient between spectral coefficients of vorticity and moisture averaged along circular rings in wavenumber space. The simulations correspond to a passive case $(\mathcal{L}=0)$ with no precipitation nor evaporation, and $\mathcal{B}=0.78$ (continuous curve), $\mathcal{B}=0.5$ (dashed curve), and $\mathcal{B}=0.25$ (dotted-dashed curve).

be of comparable accuracy when $C \neq 0$ as well (not shown).

To understand why this relation continues to hold in the turbulent flow, note that in the absence of dissipation, if we replaced $d_{2}$ in the above derivation with the full advection

$$
D_{2} \equiv \frac{\partial}{\partial t}-\frac{1}{2} \frac{\partial}{\partial x}+J\left(\psi_{2},\right)
$$

then, just as before, we can derive that

$$
D_{2} \xi=0 \text {, }
$$

with $\xi$ defined by (8). If the dissipation operator were identical for each variable, and linear, we could then prove that the dissipation damps the variance of $\xi$. There being no source of variance, $\xi$ would tend to zero and we could derive precisely the same relation between moisture and PV fluxes as for linear unstable modes. In the presence of dissipation of different forms for different variables (in particular, with Ekman damping of the lower-layer vorticity), this result is no longer exact, but we still expect it to hold as long as $\xi$ cascades to small scales and most of the dissipation of $\xi$ occurs on scales that are smaller than those responsible for the bulk of the flux.

The nondimensional temperature (or thickness) flux is equal to the PV flux, or to $(\mathcal{B}-1) \mathcal{D}_{2}$, in this homogeneous system. Therefore, we have for the total moisture transport,

$$
\left\langle v_{2} m\right\rangle=(\mathcal{B}+\mathcal{C}) \mathcal{D}_{2} .
$$

As long as $C \neq 0$, one might be tempted to define a moisture diffusivity as $\left\langle\boldsymbol{v}_{2} m\right\rangle / \mathcal{C}$. This diffusivity would be larger than PV diffusivity for $C>0$ [by a factor $(1+\mathcal{B} / \mathcal{C})]$. An analogous situation arises in continuous systems in which there is a diffusivity tensor $\mathbf{K}$ that results in meridional transport of a scalar that has contributions proportional to both the horizontal and vertical gradients of the tracer, that is,

$$
\left\langle v^{\prime} c^{\prime}\right\rangle=-K_{y y} \partial_{y} \bar{c}-K_{y z} \partial_{z} \bar{c} .
$$

The off-diagonal component $K_{y z}$ results from correlations between eddy meridional and vertical motions (e.g., Plumb and Mahlman 1987). The $\beta$ effect, as measured by $\mathcal{B}$ here, seems to control the strength of this correlation.

Another important consequence of the conservation equation for $\xi$ is that moisture is strongly correlated with lower-layer vorticity at small scales. Conservation of $\xi$ intuitively implies a cascade of $\xi$ to small scales and the dissipation of its variance. Indeed, in the case without precipitation, and if the dissipation operator is identical for all variables, then $\xi$ simply tends to 0 . If the dissipation operators are different, $\xi$ still tends to be small compared with its component fields. As the cascade of temperature $\psi_{1}-\psi_{2}$ is stopped near the deformation radius in baroclinic turbulence (Larichev and Held 1995; Salmon 1980), its spectrum is steeper than that of moisture or vorticity, so there there must be a cancellation between $m$ and $(1+C) \nabla^{2} \psi_{2} /(\mathcal{B}-1)$ at small scales. This result is very similar to the thermohaline fronts in the ocean when a strong correlation between temperature and salinity gradients leads to a weak density front (see, e.g., Klein et al. 1998). In this case, the analogous quantity to $\xi$ is simply $\rho-a T-$ $b S=0$ (assuming a linear equation of state), where $\rho$ is density, $T$ is temperature, and $S$ is salinity. The conservation of this quantity (and the absence of cascade of density) implies that small scales in $T$ and $S$ must cancel each other. 
In a simulation with no precipitation and with different values of $\mathcal{B}$ (with $\mathcal{R}=0.16$ and $\mathcal{C}=0$ ), we observe indeed that the spectral components of lower-layer vorticity and moisture are strongly correlated at small scales, with a correlation close to 1 , as can be seen in Fig. 1b. We also observe that the correlation increases when $\mathcal{B}$ is decreased. This can be easily understood as the temperature contribution in $\xi$ vanishes in the limit $\mathcal{B} \rightarrow 0$ (and $\mathcal{C}=0$ ), which leads to $\xi=m+[\eta(\mathcal{B}+$ C) $\left.+\zeta_{2}(1+C)\right] /(\mathcal{B}-1) \rightarrow m-\zeta_{2} \approx 0$ for all wavenumbers. When $\mathcal{B}+\mathcal{C}$ is larger, the contribution of temperature is significant only for low wavenumbers (compared to that of vorticity), so that there is a good correlation between vorticity and moisture at wavelengths smaller than the deformation radius.

One might expect a strong correlation between lowerlayer vorticity and moisture to be related to the Ekman damping in the lower layer, with the associated vertical Ekman pumping causing convergence and moistening. However, this derivation suggests that the Ekman damping reduces what would otherwise be an even more precise correlation by creating a difference in the way that vorticity and moisture are dissipated. In our model, vertical velocities are not caused by Ekman pumping predominately but by baroclinic processes. The mechanism we highlight is more complex than would be expected. First, there is some similarity in the "sources" of moisture and vorticity because of the low-level convergence of moisture $\left(m_{0} \boldsymbol{\nabla} \cdot \mathbf{u}_{2}\right)$ and the vortex stretching of vorticity columns $\left(f_{0} \boldsymbol{\nabla} \cdot \mathbf{u}_{2}\right)$. However, the important point is that moisture and vorticity are advected by the same horizontal flow. These two facts result in the Lagrangian conservation of $\xi$, and it is the horizontal flow and the resulting cascade of $\xi$ to small scales that can efficiently create a correlation between $m$ and $\zeta_{2}$.

When precipitation is turned on, we expect the poleward flux of moisture deficit to be reduced, as the moisture deficit $m-m_{s}$ will be zero in precipitating regions. Consistent with this expectation, we find in all cases examined that (9) is an upper bound on the flux of moisture deficit. For later reference, we refer to the ratio between the actual flux of moisture deficit and that predicted by (9) as $1-\alpha_{0}$ :

$$
\left\langle v_{2}\left(m-m_{s}\right)\right\rangle=\left(1-\alpha_{0}\right) \mathcal{B}(1+\mathcal{C}) \mathcal{D}_{2} .
$$

One can think of $\alpha_{0}$ as a particular measure of the fraction of the domain that is saturated, weighted in such a way so that one can use this measure of saturation to predict the moisture transport. Also we expect the correlation between the lower-layer vorticity and moisture to persist as well. This will be discussed in sections 5 and 6.

\section{b. Strong evaporation}

A second case of importance is the situation where moisture is saturated everywhere [i.e., $m=m_{s}=C\left(\psi_{1}\right.$ $\left.-\psi_{2}\right)$ ]. This can happen for instance when the evap- oration rate is large compared to the moisture divergence (i.e., large values of $\mathcal{E}$ ). In this limit, moisture is no longer an independent variable, and the moist static energy (2) becomes

$$
\left(\frac{D_{2}}{D t}-\frac{1}{2} \partial_{x}\right)\left(\psi_{1}-\psi_{2}\right)-\frac{\partial}{\partial x} \psi_{2}=\frac{1-\mathcal{L}}{1+\mathcal{L}} \boldsymbol{\nabla} \cdot \mathbf{u}_{2} .
$$

In dimensional units, this equation is equivalent to the temperature equation in the passive case (i.e., when $\mathcal{L}$ $=0$ ) but with a modified static stability $\sigma_{\text {sat }}$,

$$
\sigma_{\mathrm{sat}}=\frac{1-\mathcal{L}}{1+\mathcal{L} \mathcal{L}} \sigma_{0}
$$

or with a modified radius of deformation $\lambda_{\text {sat }}$ varying as $\left(\sigma_{\text {sat }} / \sigma_{0}\right)^{1 / 2}$. Here, $\sigma_{0}$ is related to $\lambda_{0}$ by $\lambda_{0}^{2}=g \sigma_{0} H^{2} / f_{0}^{2}$. As moisture is present only in the lower layer, we should more precisely talk about an effective lower-layer thickness; however, we continue to use the term "effective static stability" with this in mind. The effective static stability of the moist atmosphere in this case depends on both $\mathcal{L}$ and $\mathcal{C}$ and decreases as they both increase (Zhang 1995).

Returning to the case with finite $\mathcal{E}$ and with an unsaturated atmosphere, one is led to make the hypothesis that $\mathcal{C}$ and $\mathcal{L}$ affect the flow primarily through the combination

$$
\mu_{\mathrm{sat}}=\frac{1+\mathcal{C L}}{1-\mathcal{L}} .
$$

More generally, one may guess that there is an equivalent static stability that allows one to view the eddy statistics with a dry theory, but with a reduced value (11), most likely somewhere between the dry stability and the value appropriate for the saturated case. We return to this point below.

\section{Numerical results}

To begin an exploration of the phase space, we chose to vary parameters $\mathcal{C}$ and $\mathcal{L}$ keeping other parameters constant. We have performed a few experiments with a different evaporation rate $\mathcal{E}$ and the results we present here remain qualitatively valid in that case. The only difference brought by changing $\mathcal{E}$ is the degree of saturation of the moisture field. We performed different sets of runs, two sets keeping $\mathcal{C}$ constant $(\mathcal{C}=0$ and $C$ $=2$ ) and varying $\mathcal{L}$, and another one keeping $\mathcal{L}$ constant $(\mathcal{L}=0.3)$ and varying $\mathcal{C}$, for a total of 20 simulations.

The case of moisture at saturation in section $3 \mathrm{~b}$ suggests that the dynamics might behave similarly as a function of $\mathcal{C}$ or $\mathcal{L}$ provided that $\mu_{\text {sat }}$ varies in the same way. We thus choose to plot the total kinetic energy for each experiment as a function of $\mu_{\text {sat }}$ in Fig. 2. As expected, the increase in strength of latent heat release leads to an increase in energy. However, we observe two different regimes. When the moist parameters are relatively small (i.e., $\mu_{\text {sat }}<2.5$ ), the energy increases 
(a)

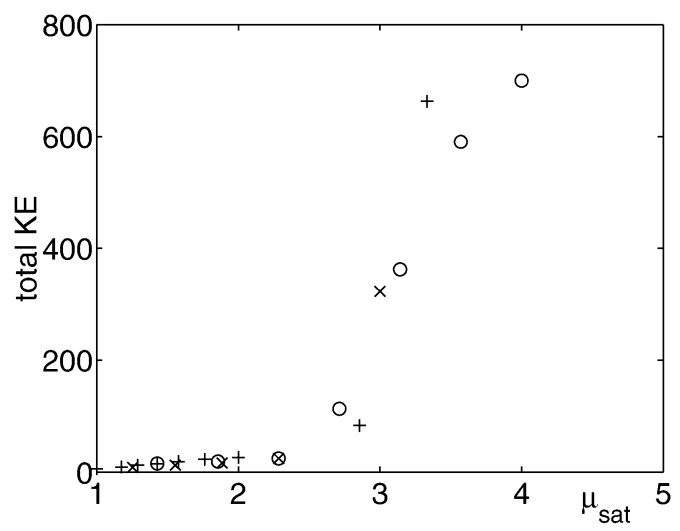

(b)

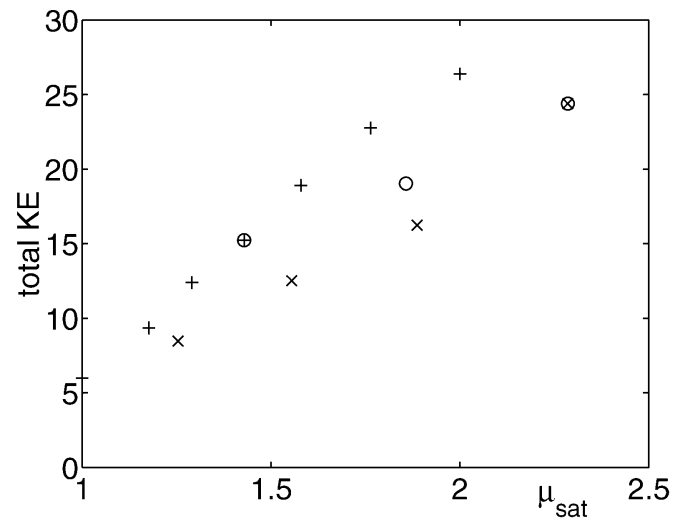

FIG. 2. (a) Total kinetic energy as a function of $\mu_{\text {sat }}$. Three different sets of runs are represented: plus signs with constant $\mathcal{C}=0$; crosses with constant $\mathcal{C}=2$; and circles with constant $\mathcal{L}=0.3$. (b) Same as (a), but for only small $\mu_{\text {sat }}$.

rather modestly - an increase by a factor of 2 in $\mu_{\text {sat }}$ leads to an increase by a factor of 4 of total kinetic energy (see Fig. 2b). We will refer to simulations with this moderate behavior as "regime I." For $\mu_{\text {sat }}>2.5$, we observe a different behavior with an explosive energy increase-an increase in $\mu_{\text {sat }}$ by a factor of 2 is accompanied by an increase in energy by a factor of 25 (see Fig. 2a). We will refer to simulations with this explosive behavior as "regime II." Simulations in regime II are also very slow to equilibrate as compared to regime I. These two regimes are also evident from other diagnostics such as vorticity skewness, as discussed in section 6 .

In an inhomogeneous environment, with a fixed amount of energy supplied to the system, the eddy energies would not grow this dramatically for the energy throughflow could not be maintained; rather, the mean state would have to find a way to adjust to prevent it from falling deep into regime II. The implication for inhomogeneous flows may be that one should expect adjustment back toward the boundary of this regime.

Concerning the dependence on $\mathcal{C}$ and $\mathcal{L}$, we see that the results in Fig. 2 do depend to a first approximation on $\mu_{\text {sat, }}$ but there is significant scatter of the different simulations in the figure. We will return to this point below.

Baroclinic turbulence on the $\beta$ plane is characterized by the presence of jets and vortices (Panetta 1993). To better understand the effect of large-scale precipitation on the storm track dynamics, it is important to examine how these coherent structures are affected by the presence of moisture. Figures $3 \mathrm{a}-\mathrm{c}$ show the total "dry" potential vorticity $\left[\left\langle q_{1}\right\rangle+q_{1}^{\prime}+(\mathcal{B}+1) y\right]$ of the upper layer, and Figs. 3d-f show the total moist PV $\left(\left\langle q_{m}\right\rangle+\right.$ $\left.q_{m}^{\prime}+\Gamma y\right)$ of the bottom layer for three different moist experiments: a "passive" run $(\mathcal{L}=0)$, a case in regime I ( $\mathcal{L}=0.3, \mathcal{C}=2$ and $\left.\mu_{\text {sat }} \approx 2.29\right)$, and one in regime II $\left(\mathcal{L}=0.3, \mathcal{C}=4\right.$ and $\left.\mu_{\text {sat }} \approx 3.14\right)$. These three cases are typical of the whole set of simulations. The passive run (Figs. 3a and 3d) represents the standard picture of baroclinic turbulence with zonal jets in the upper layer and less intense motion with a wavelike pattern in the bottom layer. When $\mathcal{C}$ or $\mathcal{L}$ is increased, the spatial organization of the PV field remains similar to the passive case (Figs. 3b and 3e), and we still observe zonal jets in the upper layer, although there is a reduction in the number of these jets (from three jets in Fig. 3a to two jets in Fig. 3b). Also, there is evidently stronger mixing in both layers as the PV contours are more convoluted. The amplitude of the lower-layer moist eddy PV rms has increased by a factor of 7.7 compared to the passive case, whereas the upper-layer eddy PV increase is much smaller (1.5). As we increase $\mu_{\text {sat }}$ further, the PV fields begin to resemble $f$-plane simulations with the disapperance of the jets and the presence of coherent vortices (Fig. 3c and 3f). The upper-layer PV contours are still oriented zonally but with large meridional excursion. The lower layer is dominated by very intense vortices, which are mostly cyclonic, and the background field is strongly homogenized.

Concerning the moisture distribution, the evaporation rate $\mathcal{E}$ was chosen such that the passive case is fairly saturated (Fig. 4a). Only regions with large downward motion have a significant moisture deficit. This happens on the eastern side of the ridges. The constant evaporation rate leads to a uniform precipitation rate equal to the evaporation rate, with only a few regions where it exceeds the evaporation rate. These latter regions concern the polar flow on the eastern side of the troughs where ascending motion is strong (not shown). Increasing $\mu_{\text {sat }}$ leads to a larger moisture deficit, which is mostly concentrated in small-scale filaments (Fig. 4b). It is also associated to a decrease in the precipitation area with a precipitation rate that greatly exceeds the evaporation rate (not shown). In regime II, the background moisture deficit is rather unsaturated (Fig. 4c). Cyclones tend to be mostly saturated whereas anticyclones have a large moisture deficit (compare with the vorticity field in Fig. $10 \mathrm{~b})$. This is consistent with argument in section $3 \mathrm{a}$ and 
(a)
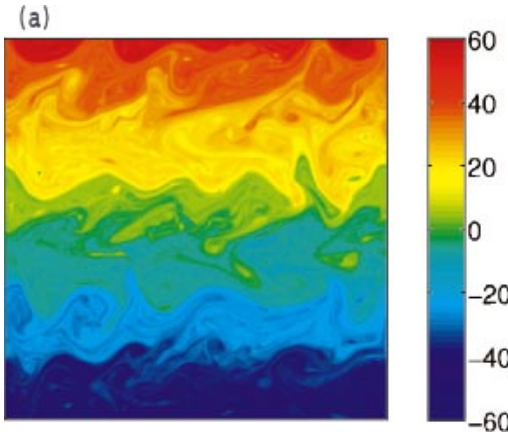

(d)
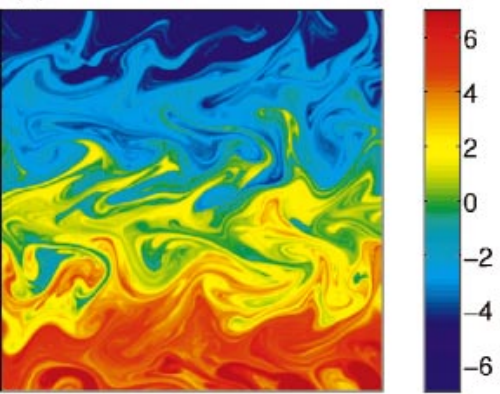

(b)

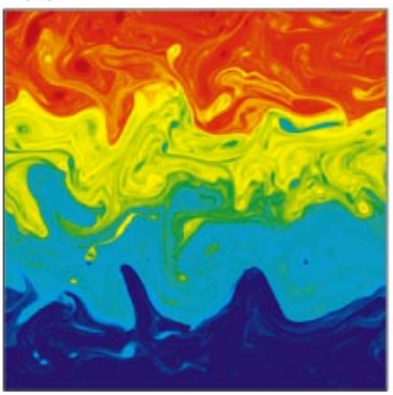

(e)

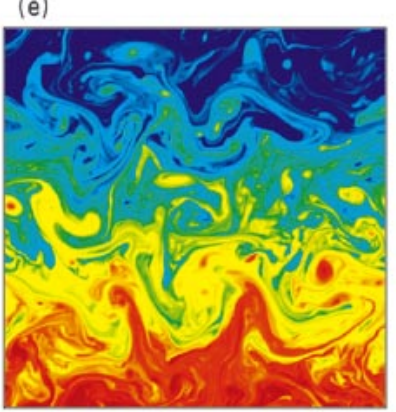

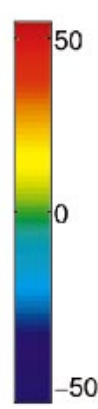

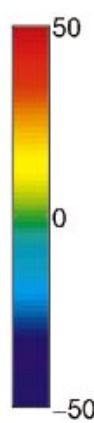

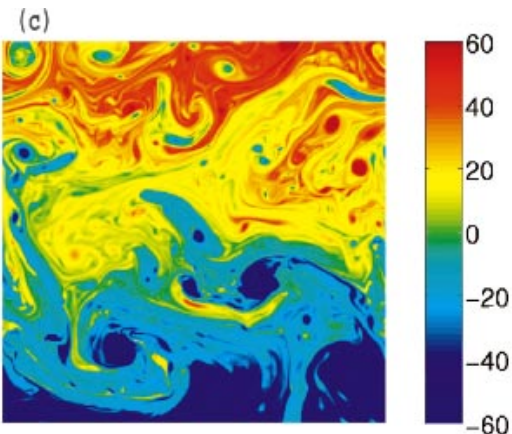

(f)

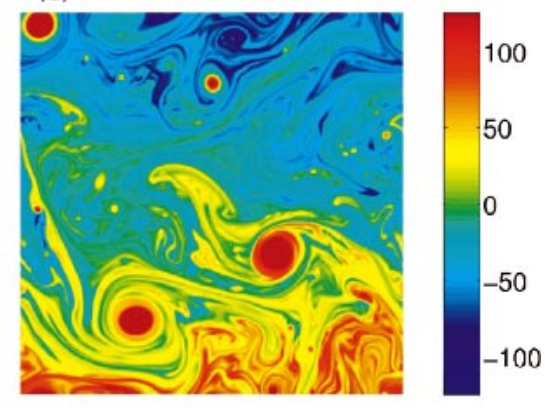

FIG. 3. Total nondimensional "dry" PV of top layer (first row) and "moist" PV of bottom layer (second row) for (a), (d) a "passive" case $(\mathcal{L}=0)$; (b), (e) a case in regime I ( $C=2, \mathcal{L}=0.3$, and $\left.\mu_{\text {sat }} \approx 2.29\right)$; and (c), (f) a case in regime II $\left(\mathcal{C}=4, \mathcal{L}=0.3\right.$, and $\left.\mu_{\text {sat }} \approx 3.14\right)$.

will be discussed in section 6 . The precipitation rate concentrates in a very small fraction of the total area with large values, mostly in ascending regions associated with cyclones (not shown).

\section{Effective static stability and energetics \\ a. Global effective static stability}

One can interpret our moist simulations by assuming that the basic effect of latent heat release is a reduction in the effective static stability of the atmosphere. This would tend to enhance the effective supercriticality of the flow and the turbulent field would behave more and more like $f$-plane turbulence. This picture is in agree- ment with the simulations as we observe a decrease in the number of jets and the appearance of vortices that eventually grow in size.

An effective dry model would not be able to explain the cyclone/anticyclone asymmetry that develops so strongly in regime II. But one would still like to determine an optimal global effective static stability, or, in our two-layer model, an effective mean thickness of the lower layer which would allow one to mimic some of the key statistics of the moist model with a dry model, especially when the latent heating is not too strong. Intuitively, one might expect this lower-layer effective static stability to be some effective weighting between saturated and unsaturated regions; that is,
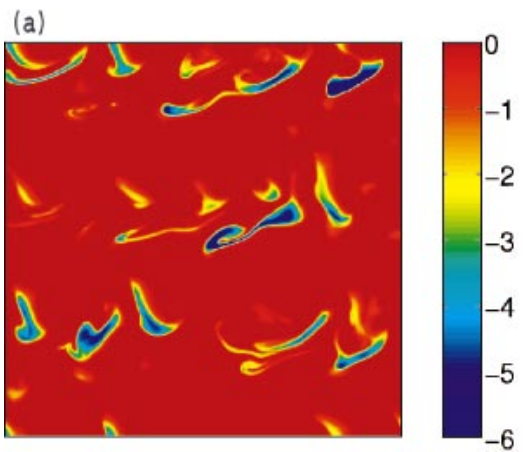

(b)

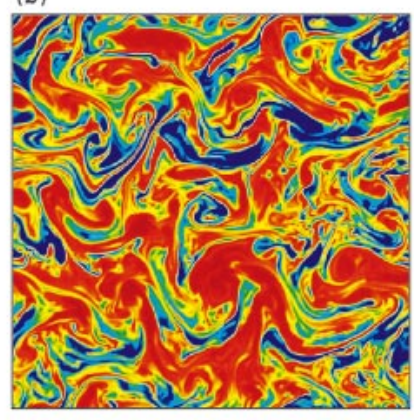

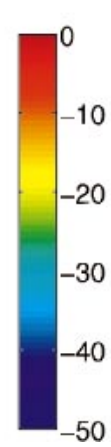

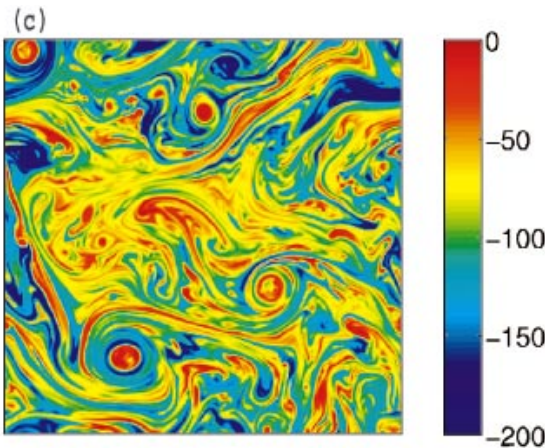

FIG. 4. Nondimensional moisture deficit for the same three cases as Fig. 3. 
(a)

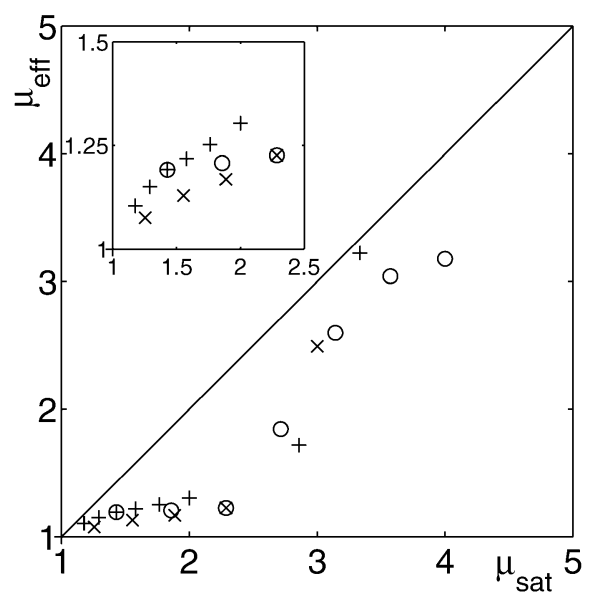

(b)

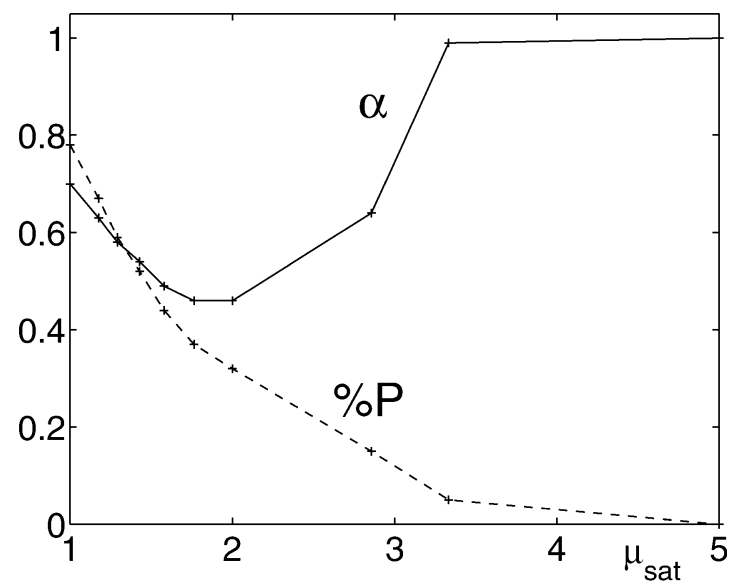

FIG. 5. (a) Nondimensional effective static stability $\mu_{\text {eff }}$ as a function of $\mu_{\text {sat }}$. The continuous curve corresponds to $\mu_{\text {sat }}$. A zoom is shown in the insert. Symbols are the same as in Fig. 2. (b) Pseudoprecipitation fraction $\alpha$ (continuous curve) and precipitation fraction (dashed curve) as a function of $\mu_{\text {sat }}$ for set of runs with $\mathcal{C}=0$. The precipitation fraction is the time average of the fractional area in which precipitation is occurring.

$$
\sigma_{\text {eff }} \equiv \frac{\sigma_{0}}{\mu_{\text {eff }}}=\sigma_{0}\left[(1-\alpha)+\alpha\left(\frac{1-\mathcal{L}}{1+\mathcal{C L}}\right)\right],
$$

where $\alpha$ is an effective weighting coefficient. The question is then how to define $\alpha$.

When discussing the passive case, we argued that the effect of precipitation is to reduce the moisture deficit flux, and described how this moisture flux reduction can be used to define a degree of saturation, $\alpha_{0}$, in the passive case. In the nonpassive case, we propose that a natural generalization of (10) is to define $\alpha$ such that

$$
\left\langle v_{2}\left(m-m_{s}\right)\right\rangle=(1-\alpha) \mathcal{B}(1+\mathcal{C}) \mathcal{D}\left(q_{m}\right) .
$$

We use the diffusivity of moist PV instead of the dry $\mathrm{PV}$ in this equation because moist PV in many ways plays an equivalent role in the moist model, as does dry PV in the dry model; for instance, it is forced only by a large-scale mean gradient and is advected by the flow, without any production by latent heat release [see Eq. (6)]. One consistency check is that, when defined in this way, we find in all numerical cases that $0<\alpha<1$ (see Fig. 5b for confirmation).

An additional reason to use $\alpha$ defined by (13) is provided by the following manipulation, in which we show that, given the flow field in this moist model, we can define a dry PV with an effective stability given by (12) that is diffused down its gradient in the lower layer with the same diffusivity with which the moist PV is diffused. We begin with the definition of the moist PV diffusivity. Taking into account that the vorticity flux is zero in the homogeneous limit, we have

$$
\left\langle v_{2}\left(\psi_{1}-\psi_{2}+\mathcal{L} m\right)\right\rangle=-\Gamma(1-\mathcal{L}) \mathcal{D}\left(q_{m}\right),
$$

where $\Gamma$ is the gradient of the moist PV [see Eq. (7)]. Splitting $\left\langle\boldsymbol{v}_{2} m\right\rangle$ in $\left\langle\boldsymbol{v}_{2}\left(m-m_{s}\right)\right\rangle$ and $\left\langle\boldsymbol{v}_{2} m_{s}\right\rangle=$ $C\left\langle v_{2}\left(\psi_{1}-\psi_{2}\right)\right\rangle$, we can express the heat flux as

$$
\begin{aligned}
(1 & +\mathcal{C L})\left\langle v_{2}\left(\psi_{1}-\psi_{2}\right)\right\rangle \\
& =-\Gamma(1-\mathcal{L}) \mathcal{D}\left(q_{m}\right)-\mathcal{L}\left\langle v_{2}\left(m-m_{s}\right)\right\rangle .
\end{aligned}
$$

Now, we use (13) to replace the moisture deficit flux by its value dependent on $\alpha$ and $\mathcal{D}\left(q_{m}\right)$. We then find

$$
\begin{aligned}
(1+\mathcal{C L})\left\langle v_{2}\left(\psi_{1}-\psi_{2}\right)\right\rangle=- & {[\mathcal{B}(1+\mathcal{C L})-\alpha \mathcal{B} \mathcal{L}(1+\mathcal{C})} \\
- & (1+\mathcal{C} \mathcal{L})] \mathcal{D}\left(q_{m}\right)
\end{aligned}
$$

using the definition of $\Gamma$. If we replace $\mu_{\text {eff }}$ by its definition (12), we have

$$
-\frac{\mu_{\mathrm{eff}}\left\langle v_{2}\left(\psi_{1}-\psi_{2}\right)\right\rangle}{\mathcal{B}-\mu_{\mathrm{eff}}}=\mathcal{D}\left(q_{m}\right) ;
$$

so that the quantity $q_{\text {eff }}=\nabla^{2} \psi_{2}+\mu_{\text {eff }}\left(\psi_{1}-\psi_{2}\right)$ with the associated mean gradient $\partial_{y} Q_{\text {eff }}=\mathcal{B}-\mu_{\text {eff }}$ has the same diffusivity as moist PV. It can be seen as a modified PV that takes into account the reduced static stability due to moist processes. This derivation says nothing about differences in the flow between dry and moist models, but it seems to be guiding us to a plausible definition of an effective stability.

We attempt to show in the following that by using Eq. (13) to define the parameter $\alpha$ in Eq. (12), one obtains an effective static stability which, when used in a dry model, does simulate many of the eddy statistics of the moist model. But we have no theory for how $\alpha$ varies with model parameters. We simply take it from the model calculations. Despite this limitation, we think the claim that the effective stability is related to the flux of moisture deficit is important, and potentially relevant for more realistic systems.

Figure 5a shows the diagnosed values of $\mu_{\text {eff }}$ as a function of $\mu_{\text {sat }}$. Consistent with other diagnostics, we observe two different regimes: for small latent heat parameters, $\mu_{\text {eff }}$ increases slowly, but for $\mu_{\text {sat }}>2.5$, the 
(a)

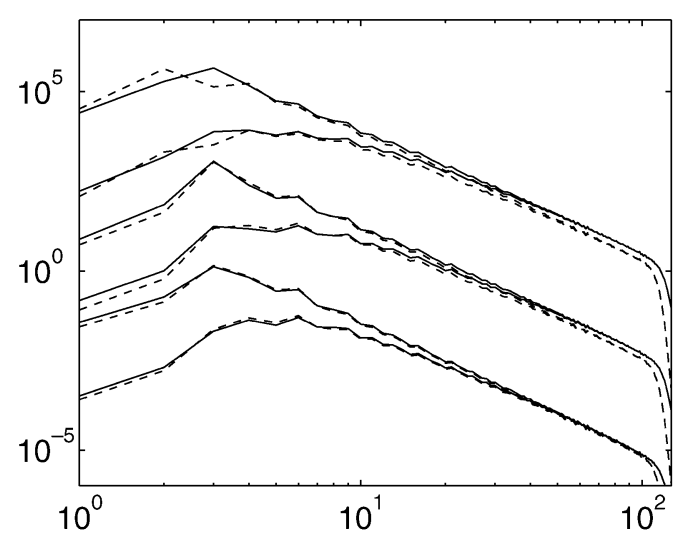

(b)

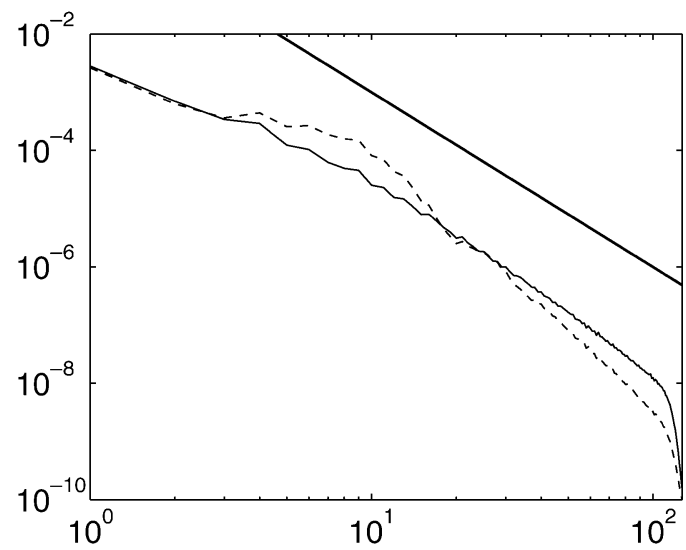

FIG. 6. (a) Nondimensional barotropic and baroclinic energy spectra for moist simulations (continuous curve) and equivalent dry simulations (dashed curve) with $\mu_{\text {eff }}=1.11,1.22,1.39$, and $\mu_{\text {sat }}=1.18,1.58,2.29$ (respectively, lower to upper curves). (b) Kinetic energy spectra of top layer (continuous) and of bottom layer (dashed) for a case in regime II $\left(C=4\right.$ and $\mathcal{L}=0.3$, such that $\left.\mu_{\text {sat }}=3.14\right)$. The bold curve corresponds to the regime in $k^{-3}$.

rise is very sudden and rapid. The evolution of the saturation fraction $\alpha$ can help explain the two regimes of evolution of $\mu_{\text {eff }}$ as a function of $\mu_{\text {sat }}$. First, $\alpha$ tends to decrease when $\mu_{\text {sat }}$ increases in the range $\mu_{\text {sat }}<2$ (Fig. $5 b)$. This is presumably due to the decrease in precipitation area as can be seen by its evolution as a function of $\mu_{\mathrm{sat}}$. Then, $\alpha$ increases and saturates toward 1 in regime II. Evidently, $\alpha$ feels only the strongest cyclonic eddies in this regime and these eddies tend to be saturated in moisture, whereas the background flow is not dynamically important in regards to the transport of moisture. Writing $\mu_{\mathrm{eff}}$ as a function of $\mu_{\text {sat }}$, we obtain

$$
1-\mu_{\text {eff }}^{-1}=\alpha\left(1-\mu_{\text {sat }}^{-1}\right),
$$

which shows that the observed decrease of $\alpha$ when $C$ and $\mathcal{L}$ increase counterbalances the increase of $1-$ $\mu_{\text {sat }}^{-1}$ when $\mu_{\text {sat }}$ increases, so that $\mu_{\text {eff }}$ increases slowly as is observed. On the other hand, when $\alpha$ increases, the two effects add to each other and $\mu_{\text {eff }}$ can increase rapidly. Because $\mu_{\text {eff }}$ controls the effective supercriticality of the flow $\chi_{\text {eff }}=\mu_{\text {eff }} / \mathcal{B}$, it should have a direct impact on the energetics and its slow/rapid growth could explain the observed abrupt transition between the two dynamical regimes. On the $f$ plane, there would not be any meridional flux of moisture deficit because of (13). In that case, one would need a different relation to define $\alpha$ and an effective static stability.

To illustrate the usefulness of the effective static stability, one can compare the energy spectra obtained in the moist model and in a equivalent dry model. The dry model uses a reduced deformation radius only in the lower layer. Consistent with the discussion above, we find that this results in a better fit to the moist results than if we modified the upper-level radius of deformation as well. Figure $6 \mathrm{a}$ shows the baroclinic and barotropic kinetic energy spectra nondimensioned by $\varepsilon^{2 / 3}$, where $\varepsilon$ is the dissipation of barotropic energy. The reason of this choice comes from the inverse cascade that should imply standard spectra of the form $\varepsilon^{2 / 3} k^{-5 / 3}$ (for the barotropic energy). The dry equivalent model seems to be in agreement with the moist model for simulations in regime I. In regime II, we found that the dry model fails to reproduce the modal energy spectra of the moist simulations (not shown). Very energetic coherent vortices in these simulations create an energy peak at their scales (see the "bump" in the bottom-layer energy spectrum at $k \approx 10$ in Fig. 6b). An equivalent dry model would not possess such coherent vortices that dominate the flow energetics.

Another test for our estimation of $\mu_{\text {eff }}$ is to see if the statistics of the moist model are a well-defined function of $\mu_{\text {eff }}$ and if they scatter less than with $\mu_{\text {sat }}$. Figure 7 displays the total kinetic energy as a function of $\mu_{\text {eff }}$. The data scatter less if compared to Fig. 2b, which uses $\mu_{\text {sat }}$ (Fig. 7b). Also, we do not see a knee when passing from regime I to regime II (Fig. 7a) which could indicate that the properties of the two regimes are not so statistically different when viewed from this perspective.

The effective static stability we introduced should only affect the lower layer since our argument is based on the lower-layer moisture. What happens in the upper layer? We observe that the vortex asymmetry does not develop in the upper layer (see Fig. 11) and the kinetic energy spectrum in this layer stays similar to the dry case (see Fig. 6b). Therefore the upper-layer eddy statistics seem much less affected by latent heat release than in the lower layer, despite the fact that both layers are forced equally by the latent heat release.

\section{b. Eddy fluxes and energetics}

A final test for our prediction of the global static stability is to extend the flux closure theory developed in Lapeyre and Held (2003) for the dry model to this moist 
(a)

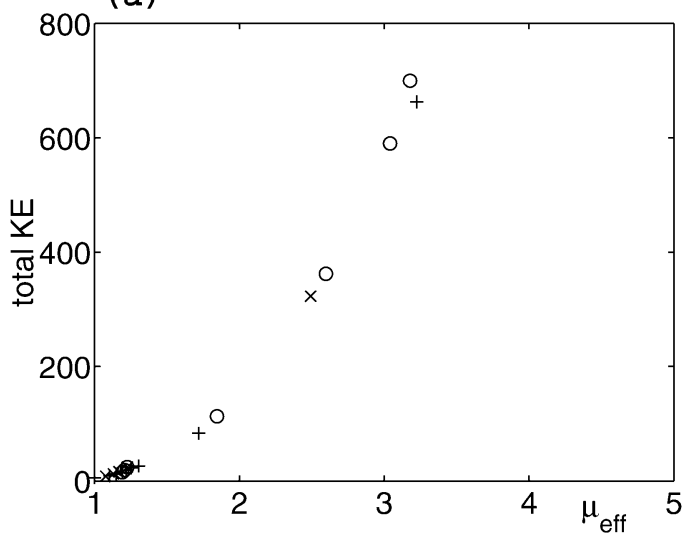

(b)

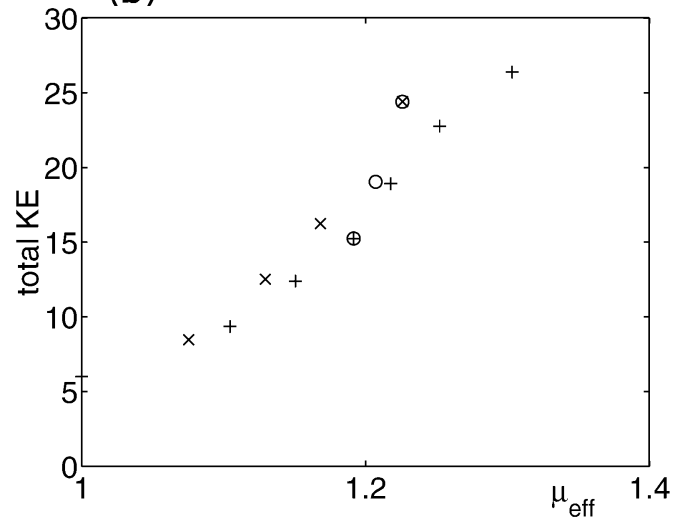

FIG. 7. (a) Total kinetic energy as a function of $\mu_{\text {eff }}$. (b) Same as (a) but for only small $\mu_{\text {eff }}$. Symbols are the same as in Fig. 2.

case. Since we have no theory for $\alpha$, this is a test of consistency, rather than a complete theory. In the dry theory, one starting point is a relationship between the barotropic energy dissipation rate $\varepsilon$ and diffusivity $\mathcal{D}$ :

$$
\mathcal{D} \propto \varepsilon^{3 / 5} \beta^{-4 / 5}
$$

(see also Barry et al. 2002). The lower-layer PV diffusivity is found to obey the relation (14) quite well in the dry model. Once again substituting moist PV for dry PV in this moist model, we obtain

$$
\mathcal{D}_{m} \propto \varepsilon^{3 / 5} \beta^{-4 / 5} .
$$

Figure $8 \mathrm{a}$ shows that this relation is valid in regime I (corresponding to diffusivities smaller than $4 U \lambda$ ) whereas the prediction underestimates the actual diffusivity in regime II. In the dry model, we have shown that (14) can be explained through the inverse cascade of barotropic energy on the $\beta$ plane (Lapeyre and Held 2003): the rate of energy that is cascading to small scales $\varepsilon$

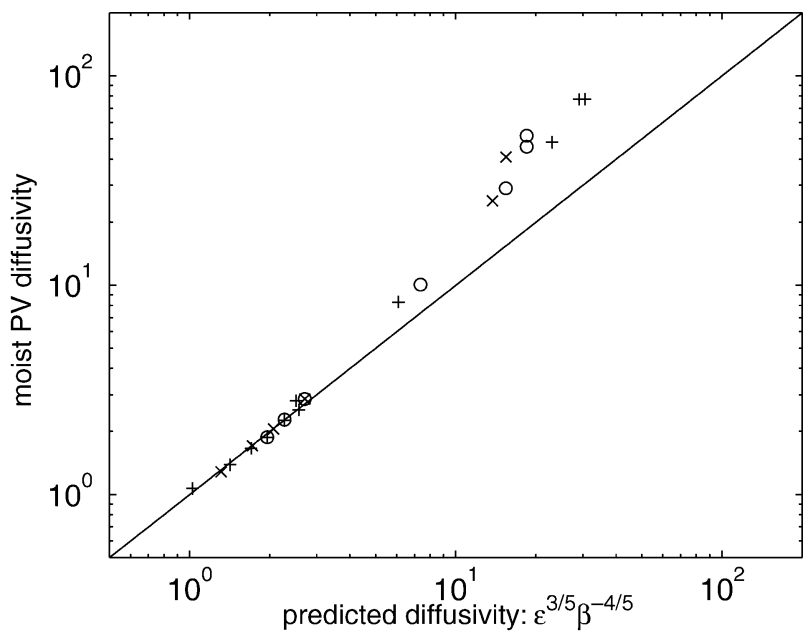

FIG. 8. (a) Moist diffusivity as a function of prediction $c \varepsilon^{3 / 5} \beta^{-4 / 5}$ with $c=1.7$ and diffusivities nondimensioned by $U \lambda_{0}$. Symbols are the same as in Fig. 2. and the $\beta$ effect set the properties of the dominant eddies, in particular their length and velocity scales. Experimentally, the lower-layer PV seems to be diffused by these eddies, so that its diffusivity depends only on $\varepsilon$ and $\beta$. In the moist model, an analogous scenario is at play, in that the essence of the barotropic cascade is unaffected by latent heat release, which is dominant at smaller scales with relatively larger divergences (which are below the effective deformation radius scale). Here, we need to diffuse the moist PV because it is forced only by a large-scale mean gradient in the same way as the dry PV of the dry model. As the properties of regime I are similar to classic baroclinic turbulence, (15) should apply as observed. Moreover, in regime II, coherent vortices dominate the lower-layer flow, so that we cannot use the analogy with the dry theory anymore.

\section{c. Available potential energy}

If one had a theory for $\varepsilon$ as a function of the parameters $(\mathcal{B}, \mathcal{C}, \mathcal{L})$ and, possibly, the diffusivity $\mathcal{D}$, one could then predict the diffusivity from (15), as in the theory of Lapeyre and Held (2003) or Barry et al. (2002), without having to determine an effective static stability. Lapeyre and Held (2003) (following Held and Larichev 1996) relate $\varepsilon$ to $\mathcal{D}$ and $\mathcal{B}$ by examining the generation of available potential energy (APE) while Barry et al. (2002) invoke an entropy budget. It is instructive to examine the generation of available potential energy in this moist model in an attempt to relate $\varepsilon$ to $\mathcal{D}$. We will see that the result resembles a moist entropy budget.

From the interface equation, one finds

$$
\frac{\partial}{\partial t} \mathrm{APE}=\mathscr{P}_{d}+\mathcal{H}-\varepsilon
$$

where APE $=\left\langle\eta^{2}\right\rangle / 2, \mathscr{P}_{d}=\left\langle v_{2} q_{2}\right\rangle, \varepsilon$ equals to the transfer to kinetic energy $\left(\left\langle\eta \boldsymbol{\nabla} \cdot \mathbf{u}_{2}\right\rangle\right)$, and $\mathcal{H}$ is the production of APE by latent heat release $[\mathcal{L}\langle(P-E) \eta\rangle]$. We assumed that the small-scale diffusion of temperature to 
be negligible. As we can see, there are two key complications in the moist model. First, the eddy APE is directly generated by latent heat. This source would have to be estimated in order to derive a closed theory for the moist PV diffusivity in (15). Second, the potential vorticity $q_{2}$ is no longer conserved in the presence of latent heating, so we cannot think of its flux as diffusive. It is thus more attractive to use the moist static energy as a point of departure for a moist available potential energy; that is,

$$
\begin{aligned}
\frac{1}{2} \partial_{t}\left\langle(\eta+\mathcal{L} m)^{2}\right\rangle= & (1-\mathcal{L})\left\langle(\eta+\mathcal{L} m) \boldsymbol{\nabla} \cdot \mathbf{u}_{2}\right\rangle \\
& +(1+\mathcal{C L})\left\langle\boldsymbol{v}_{2}(\eta+\mathcal{L} m)\right\rangle \\
& +\mathcal{L}\left\langle F_{m}(\eta+\mathcal{L} m)\right\rangle .
\end{aligned}
$$

This equation has the advantage that it does not involve $P$ explicitly, but we cannot simply combine this equation with the kinetic energy equation, since $\left\langle(\eta+\mathcal{L} m) \boldsymbol{\nabla} \cdot \mathbf{u}_{2}\right\rangle$ rather than $\left\langle\eta \nabla \cdot \mathbf{u}_{2}\right\rangle$ appears on the rhs. One can remedy this situation by using the moisture deficit variance budget:

$$
\begin{aligned}
\frac{1}{2} \partial_{t}\left\langle\left(m-m_{s}\right)^{2}\right\rangle= & -(1+\mathcal{C})\left\langle(m-C \eta) \boldsymbol{\nabla} \cdot \mathbf{u}_{2}\right\rangle \\
& +(1+\mathcal{L} \mathcal{L})\left\langle(E-P)\left(m-m_{s}\right)\right\rangle \\
& +\left\langle F_{m}\left(m-m_{s}\right)\right\rangle,
\end{aligned}
$$

where $F_{m}$ corresponds to the small-scale dissipation of moisture. We can combine (16) and (17) to eliminate $\left\langle m \boldsymbol{\nabla} \cdot \mathbf{u}_{2}\right\rangle$ in the equations; that is,

$$
\begin{aligned}
\frac{1}{2} \partial_{t}[ & \left.\mathcal{L}(1-\mathcal{L})\left\langle d^{2}\right\rangle+(1+\mathcal{C})\left\langle(\eta+\mathcal{L} m)^{2}\right\rangle\right] \\
= & (1-\mathcal{L})(1+\mathcal{C})(1+\mathcal{C} \mathcal{L})\left\langle\eta \nabla \cdot \mathbf{u}_{2}\right\rangle \\
& +(1+\mathcal{C})(1+\mathcal{C} \mathcal{L})\left\langle v_{2}(\eta+\mathcal{L} m)\right\rangle \\
& +(1+\mathcal{C L})(1-\mathcal{L}) \mathcal{L}\langle(E-P) d\rangle \\
& +\mathcal{L}(1+\mathcal{L} \mathcal{L})\left\langle F_{m}(m+\eta)\right\rangle,
\end{aligned}
$$

where $d=m-m_{s}$ is a moisture deficit variable. Finally, we obtain a budget in terms of the variance of $d$ and $\eta$ $+\mathcal{L} m$, such that

$$
\begin{gathered}
\frac{1}{2} \partial_{t}\left[\frac{\mathcal{L}\left\langle d^{2}\right\rangle}{(1+\mathcal{C L})(1+\mathcal{C})}+\frac{\left\langle(\eta+\mathcal{L} m)^{2}\right\rangle}{(1-\mathcal{L})(1+\mathcal{C})}\right] \\
=\left\langle\eta \nabla \cdot \mathbf{u}_{2}\right\rangle+\left\langle v_{2} q_{m}\right\rangle \\
\quad+\frac{\mathcal{L}\langle(E-P) d\rangle}{1+\mathcal{C}}+\frac{\mathcal{L}\left\langle m F_{m}\right\rangle}{(1-\mathcal{L})(1+\mathcal{C})},
\end{gathered}
$$

where we assume that $\left\langle F_{m} \eta\right\rangle$ can be neglected compared to $\left\langle F_{m} m\right\rangle$. (The spectrum of the dissipation of moisture is dominated by the small scales of the moisture itself; the temperature has little variance on small scales, so the correlation $\left\langle F_{m} \eta\right\rangle$ is very small.) Also we replace the moist static energy flux by the moist PV flux as vorticity does not contribute to this flux in the homogeneous limit. If we assume that precipitation only occurs when the flow is saturated, then $\langle P d\rangle=0$ and $P$ no longer appears on the rhs. A similar derivation using the variance budget for $m$ itself rather than $d$ would result in the reappearance of $P$ on the rhs.

Referring to the quantity in brackets on the lhs of (18) as a moist available potential energy (MAPE) and combining with the kinetic energy equation as before, we have

$$
\frac{\partial}{\partial t} \mathrm{MAPE}=\mathcal{P}-\mathcal{N}-\mathcal{S}-\varepsilon
$$

where we refer to

$$
P \equiv\left\langle v_{2} q_{m}\right\rangle
$$

as the production, and

$$
\mathcal{N} \equiv-\langle E d\rangle
$$

as the dehumidification. Here, $\mathcal{S}$ is the small-scale diffusion. In a steady state,

$$
\varepsilon=\mathcal{P}-\mathcal{N}-\mathcal{S},
$$

and $\varepsilon$ will be balanced by frictional dissipation in the kinetic energy budget (i.e., the energy dissipation rate $\varepsilon)$. Since $d<0, \mathcal{N}$ is positive. It acts to decrease the moisture deficit variance [the term "dehumidification" follows the usage in Pauluis and Held (2002)]. The effects of small-scale mixing are to destroy moisture variance, so we can also assume that $S>0$. Therefore, we expect that the presence of $\mathcal{N}$ and $\mathcal{S}$ will reduce the value of the frictional dissipation $\varepsilon$ beneath that predicted from $\mathcal{P}$ alone. This relation is striking in its similarity to that discussed in the context of moist convection by Pauluis and Held (2002), in which the thermodynamic efficiency is also reduced by evaporation into unsaturated air and by diffusion of moisture. There is clearly a connection between an entropy budget of the sort discussed by Rennó and Ingersoll (1996), Emanuel and Bister (1996), and Pauluis and Held (2002), and a moist available potential energy budget of the sort attempted here, but we do not understand this connection fully. Similar arguments can be obtained in the context of a QG model continuous in the vertical.

The MAPE budget will serve to illustrate the difficulties that arise when trying to develop a theory for the eddy heat flux in the moist context. As discussed above, one can relate the moist PV diffusivity to the energy dissipation $\varepsilon$. To close the problem, one needs another relation between $\varepsilon$ and the eddy heat flux. Figure 9 shows the standard available potential energy budget of the model, as a function of $\mu_{\text {sat }}$, holding $C=0$. Dissipation $\varepsilon$ was evaluated by using the frictional dissipation of barotropic energy (the results are qualitatively similar if we use total energy dissipation instead). The production of energy by latent heat release $\mathcal{L}(P-E)$ increases moderately compared to the production by dry 


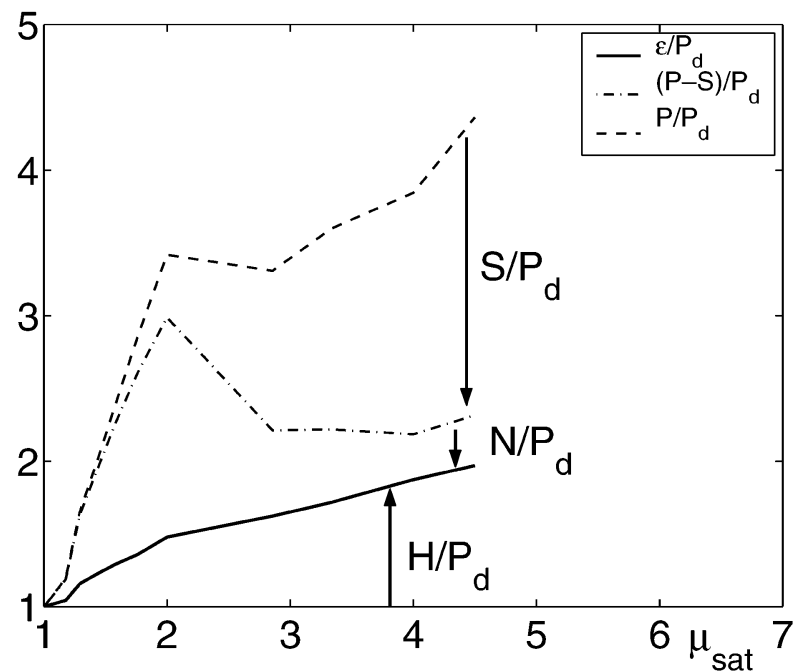

FIG. 9. Total energy production $\varepsilon / \mathscr{P}_{d}$ as a function of $\mu_{\text {sat }}$ (continuous curve), and production due to the moist static energy flux $\mathscr{P} / \mathcal{P}_{d}$ (dashed curve). The dashed-dotted curve represents the relative production due to moist static energy and moisture diffusion [i.e., $(\mathscr{P}-S) / \mathscr{P}_{d}$ ]. The different arrows symbolize the magnitude of the energy production due to latent heat release $\mathcal{H} / \mathcal{P}_{d}$ and the energy destruction by diffusion of moisture $\mathcal{S} / \mathscr{P}_{d}$ and dehumidification $\mathcal{N} / \mathscr{P}_{d}$. This corresponds to the set of simulation with $C=0$.

PV transport when increasing the latent heating parameter $\mu_{\text {sat }}$, It represents only $50 \%$ on the total energy production $\varepsilon$. If now we use the MAPE budget (19), the energy production by enthalpy transport $P$ increases roughly linearly with $\mu_{\text {sat }}$. To balance this large energy source, there is a large energy sink by dehumidification $\mathcal{N}$ and moisture diffusion $\mathcal{S}$. For small convective parameters, the principal sink comes from the dehumidification. This is due to our choice of the basic state (i.e., when $L=0$ ) with a moisture field almost saturated everywhere. When the convective parameters are larger, the moisture diffusion becomes the main contributor to the reduction in the energy generation rate. Most of the production by moist static energy transport is used to counteract the dehumidification and the moisture diffusion.

This process shows that despite a large latent heat energy available for the total energy, the model extracts only a small portion of this energy, while the remaining serves to balance the moist processes (dehumidification plus diffusion). Thus, there is a high inefficiency of the heat engine, which is unable to convert the latent heat energy into kinetic energy, as explained by Pauluis and Held (2002).

\section{Cyclone/anticyclone asymmetry}

The moist simulations develop a strong asymmetry between cyclones and anticyclones despite the quasigeostrophic formalism. In the passive case, cyclones and anticyclones have similar properties (amplitude, dynamics, ... ) because of the internal symmetry of the equations. [See Arbic and Flierl (2001) for an example of a dry QG model with mean easterlies that lead to a cyclone/anticyclone asymmetry.] In the presence of latent heat release, the asymmetry toward stronger cyclones is expected since it is well known that latent heating intensifies cyclonic development near the surface (Emanuel et al. 1987; Stoelinga 1996; Whitaker and Davis 1994). However, the characteristics of vortices in regime II are somewhat different from the ones we might intuitively expect. Therefore, it is useful to examine in details their properties in a similar manner to Polvani et al. (1994) and Legg and McWilliams (2001) in other situations.

The temperature $\psi_{1}-\psi_{2}$ associated with the cyclones is strongly positive as expected since latent heat release (associated with precipitation) increases temperature. However, despite this baroclinic forcing, strong vortices have the same sign of vorticity in each layer (see Fig. 10): bottom-layer cyclones are generally cyclonic in vorticity in the upper layer, but with a much smaller (a)

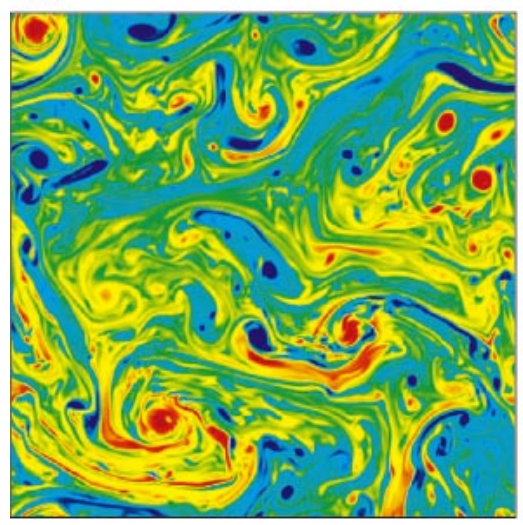

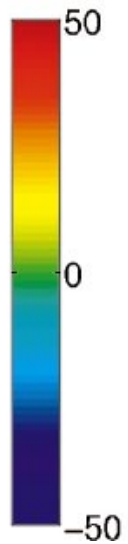

(b)

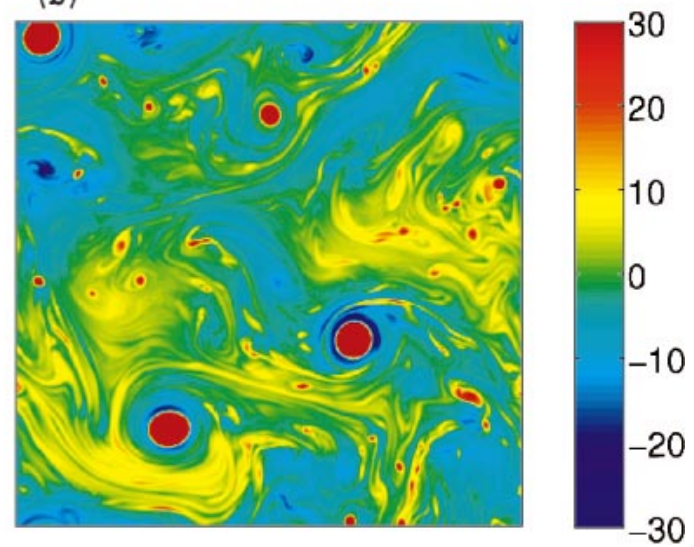

FIG. 10 . Vorticity field for a case in regime II (i.e., $\mu_{\text {sat }} \approx 3.03$ ): (a) top layer; (b) bottom layer. The square root of the amplitude is shown in order to highlight the vorticity sign. 
(a)

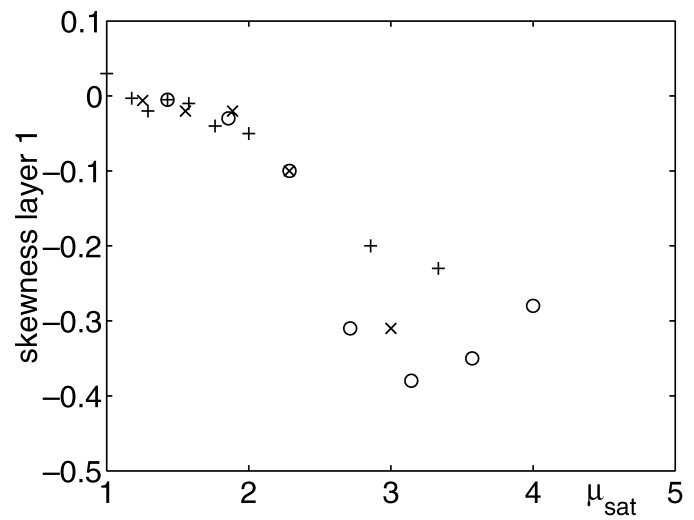

(b)

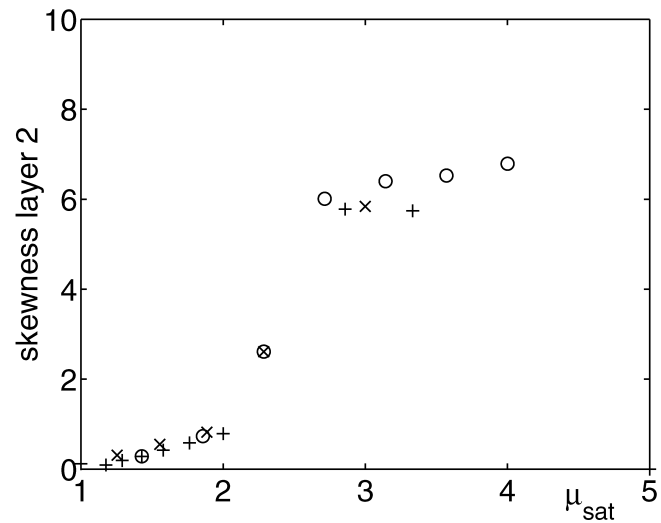

FIG. 11. Vorticity skewness $\left\langle\omega^{3}\right\rangle /\left(\left\langle\omega^{2}\right\rangle\right)^{3 / 2}$ for (a) upper layer and (b) bottom layer as a function of $\mu_{\text {sat }}$. Symbols are the same as in Fig. 2.

magnitude. In contrast, weak bottom-layer anticyclones are associated with stronger anticyclones in the upper layer, consistent with the equivalent barotropic character of the dry model. Starting from a developed dry flow and suddenly turning on latent heat release, we observe a development of small-scale filaments of opposite signs in vorticity in each layer. These filaments are dynamically unstable and roll into vortices. Thus, the forcing is clearly baroclinic but, as the flow equilibrates, vortices tend to barotropize through baroclinic processes.

A natural way to quantify the cyclone/anticyclone asymmetry is to compute the vorticity skewness $\left\langle\omega^{3}\right\rangle /\left\langle\omega^{2}\right\rangle^{3 / 2}$ for both layers. Figure 11 shows that the asymmetry develops steadily in both layers for regime I $\left(\mu_{\text {sat }}<2.5\right)$. The upper layer has a weak tendency toward anticyclones, whereas the lower layer displays a strong tendency toward cyclones. When $\mu_{\text {sat }}$ is increased further, a sharp transition occurs and the skewness saturates around 6-7 in the lower layer for regime II. In the upper layer, the skewness remains quite small, toward anticyclones. This diagnostic is consistent with the vorticity spatial field (Fig. 10) as the asymmetry between cyclones and anticyclones is discernible only in the lower layer. In the upper layer, the vortices are less dominant as the vorticity kurtosis $\left(\left\langle\omega^{4}\right\rangle /\left\langle\omega^{2}\right\rangle^{2}\right)$ is of order 10 there, whereas it is around 50 in the lower layer. This situation contrasts with the passive case and even with regime I as the kurtosis is around 3.6 in the upper layer and 3.4 in the lower one for the passive case and only a bit larger for runs in regime I. Also, the kinetic energy spectra (Fig. 6b) are consistent with the vorticity skewness as we see that bottom-layer vortices are associated with a bump in energy between wavenumbers 3 and 20 near the dry deformation radius $\left(k_{d}=9\right)$ and a very steep slope (close to $k^{-4.5}$ ) for high wavenumbers. By contrast, the upper-layer kinetic energy has a standard slope (close to $k^{-3}$ ) as the vortices do not dominate the flow there.

Vortices are able to develop in the lower layer despite the presence of friction in this layer. Actually the lowerlayer kinetic energy is even larger than the upper-layer energy in regime II, whereas it is the opposite in regime I. Danilov and Gurarie (2001) showed that for the onelayer problem on the $f$ plane, linear friction prevents the appearance of coherent vortices, and this is also the case for the bottom layer of our passive simulation (see Fig. 3d). In the moist case, the additional forcing by latent heat release thus seems to add enough energy so that vortices are able to persist, even in presence of bottom friction. We performed some simulations starting with initial conditions in regime II but with smaller values of $\mathcal{C}$ and $\mathcal{L}$ (corresponding to regime I) to see if these vortices are able to persist in a less favorable environment. We found that they decay and disappear; vortices require strong latent heating to persist in these simulations.

The existence of such cyclones can be related to dynamical feedback between moisture and vorticity in the bottom layer. As explained in section 3a, there is a strong correlation between the lower-layer vorticity and moisture through their conservation properties. This correlation is responsible of the relatively moist character of cyclones and the relatively dry character of anticyclones. This has the consequence that cyclones are able to intensify themselves through latent heat release since their moisture content is near saturation. Such an effect is absent for the anticyclones since they are depleted in moisture. This feedback is likely to act only in the bottom layer because the upper layer is not subject to a correlation between vorticity and moisture. The reason is that the upper-layer vorticity is not advected by the same horizontal flow as is the low-level moisture, and this horizontal advection is central to build the correlation. This explains why the asymmetry is very strong in the lower layer and quite weak in the upper.

To verify that this mechanism is at play in our simulations, we computed the joint probability density functions (pdfs) of bottom-layer vorticity and moisture def- 
(a)

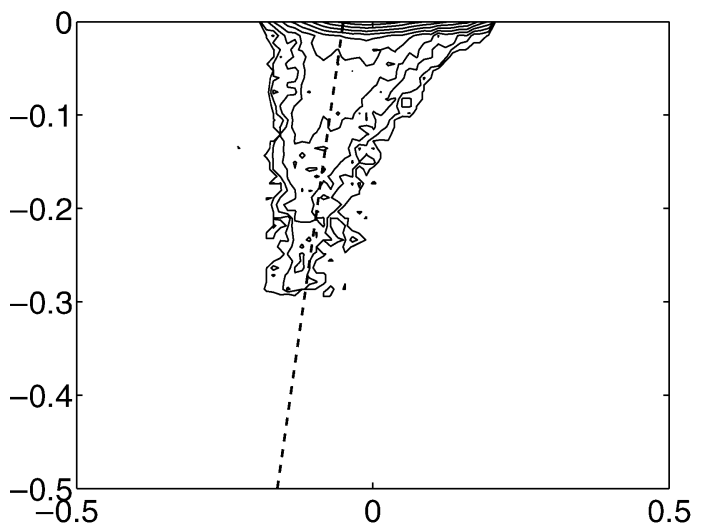

(c)

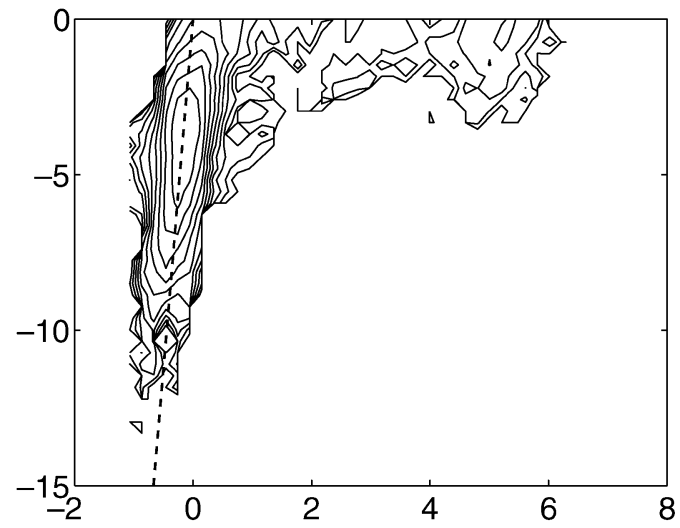

(b)

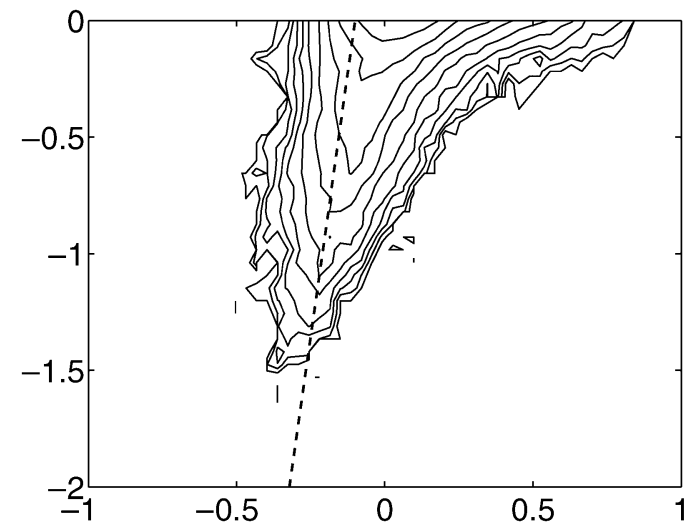

(d)

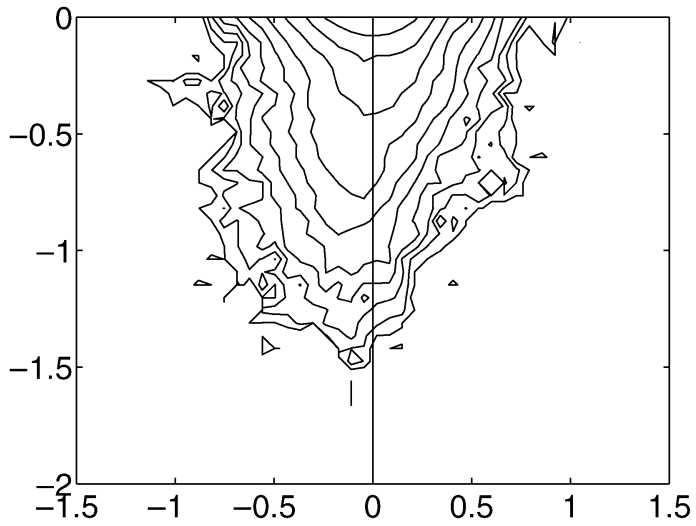

FIG. 12. Joint pdf of bottom-layer vorticity (abscissa) and moisture deficit (ordinate) in three cases: (a) passive case $\mathcal{C}=\mathcal{L}=0$, (b) case in regime I ( $C=1$ and $\mathcal{L}=0.3$ ), and (c) case in regime II ( $C=4$ and $\mathcal{L}=0.3$ ). (d) Same as (b) but with upper-layer vorticity in abscissa.

icit for different cases (Fig. 12). For the three cases (passive, regime I, and regime II), we see a clear correlation between the two fields: anticyclonic regions tend to have a large moisture deficit, whereas cyclonic regions tend to be saturated. Also for the regimes I and II, the regions at saturation have a broad range of vorticity values preferentially toward cyclonic vorticity. This is most striking for regime II, for which the joint pdf exhibits a local maximum for values of nondimensional vorticity around 5 . By contrast, moisture and upper-level vorticity (Fig. 12d) do not show a clear relationship, even if some asymmetry can be seen in the figure. Thus, it is not only the convergence motions (through vortex stretching) that are responsible for the correlation and the intensification of cyclones, but the horizontal advection plays an essential role: moisture and upper-layer vorticity are advected by different flows, which prevent their correlation. To test further if the correlation between vorticity and saturation deficit can be predicted from the passive dynamics, we also added a curve with a slope corresponding to the relation $m-m_{s}=(C+1) \nabla^{2} \psi_{2} /(1-\mathcal{B})$, setting $\eta=0$ in (8), and assuming $\xi \approx 0$. This relation corresponds to the cascade of $m-\nabla^{2} \psi_{2}+(\mathcal{B}+\mathcal{C}) q_{2} /(1-\mathcal{B})$ to small scales and neglects small-scale temperature $\psi_{1}-\psi_{2}$. We see that there is some tendency in the pdfs to follow this relationship but it is very qualitative due to the role of precipitation in destroying the correlation.

\section{Conclusions}

In this paper, we have examined moist baroclinic turbulence in the idealized context of the homogeneous quasigeostrophic two-layer model. We have included moist processes through an explicit moisture equation in the lower layer and with a simple precipitation scheme. Two key parameters (the coefficient of linearization of the Clausius-Clapeyron relationship $C$ and the strength of latent heating $\mathcal{L}$ ) control the moist baroclinic dynamics.

We find the existence of two dynamical regimes when increasing $C$ and $\mathcal{L}$ : one of moderate increase of energy with a turbulent field similar to dry baroclinic turbulence, with weak asymmetry between cyclones and an- 
ticyclones in the lower; the other regime containing very intense cyclones in the bottom layer. These cyclones have a warm core but have the same sign of vorticity in both layers despite baroclinic latent heat forcing. We also observe anticyclones intensified in the upper layer but they tend to be less energetic than their cyclonic counterparts.

We proposed some interpretation of the mechanisms involved in these simulations. Different diagnostics showed that the moderate regime of moist turbulence behaves as if its global static stability is decreased, which in turn increases the supercriticality of the flow. We have suggested a way of determining a posteriori a global effective static stability and show that it is decreasing with increasing $\mathcal{L}$ and $\mathcal{C}$. We have provided some evidence that the quantity we introduce is a good diagnostic of the effective static stability. We have also provided some mechanisms to explain the asymmetry between cyclones and anticyclones. We showed there is likely a dynamical feedback between moisture and vorticity that intensifies the bottom-layer vortices: the transport of moisture and vorticity both horizontally and vertically implies a correlation between the two variables, which promotes latent heat release inside cyclones. In our mechanism, the horizontal transport is essential to set this correlation: even if the low-level moisture convergence is coupled to upper-layer vortex stretching (by the interface displacement), the fact that upper-layer vorticity and lower-layer moisture are advected by different flows inhibits any correlation between them. This explains why the vortex asymmetry is much weaker in the upper than in the lower layer.

We find in our model that a diffusive theory for the eddy horizontal flux of moisture using only the horizontal moisture gradient underestimates this flux. Because of correlations between horizontal and vertical motions resulting from baroclinic processes acting in the presence of the $\beta$ effect, one cannot neglect the influence of off-diagonal components of the diffusivity tensor on the moisture deficit flux. This may suggest modified approaches to moisture transport in more complex models with parameterized eddies.

We have shown that, at least, for the regime of weak latent heating, the concepts of dry baroclinic turbulence can still be applied to these moist simulations, as the flow properties are affected mainly by the variation of the effective static stability. The energy budget can be rewritten in a form that generalizes available potential energy to the moist case. It highlights the role of moisture diffusion and dehumidification of the air in reducing the efficiency of the latent heat release. This result is similar to the conclusions of Pauluis and Held (2002) obtained by an entropy budget for cumulus convection.

One can think that regime I is typical of the conditions of the earth's atmosphere since the extratropics are only weakly baroclinically unstable and latent heating does not modify too strongly the dynamics of synoptic motions. The abrupt transition from regime I to regime II as $\mathcal{C}$ and $\mathcal{L}$ are increasing in this model is striking, and suggests that an inhomogeneous system, with a finite energy supply, may have difficulty penetrating regime II. It is interesting to speculate whether more realistic models of moist atmospheres would exhibit similar behavior.

Acknowledgments. The authors would like to thank Geoff Vallis and Isidoro Orlanski for helpful discussion about this work. We also thank two anonymous referees for their careful reading of the manuscript and helpful comments. All simulations were performed on the supercomputers at NOAA's Geophysical Fluid Dynamics Laboratory, using a QG code originally written by S. Smith and G. Vallis and parallelized by GL and S. Smith. GL was funded under a grant/cooperative agreement with the National Oceanic and Atmospheric Administration (NA07RG0002).

\section{APPENDIX}

\section{Numerics}

The numerical model makes the use of an implicit exponential cutoff filter as a small-scale dissipation. This filter presents the advantage to be scale selective and not to dissipate scales larger than a certain threshold. More explicitly,

$$
F(k)= \begin{cases}\exp \left[-a\left(\frac{k-k_{\mathrm{cut}}}{k_{\max }-k_{\mathrm{cut}}}\right)^{s}\right] & \text { for } k>k_{\mathrm{cut}}, \\ 1 & \text { for } k \leq k_{\mathrm{cut}},\end{cases}
$$

with $a=\log (1+2 \pi / N)$ and $N=256$ [see Smith et al. (2002) for further discussion]. The exponent $s$ is equal to 6 , and $k_{\text {cut }}=90$ in our simulations.

Concerning the numerical scheme, there are a few issues to discuss. First, the instantaneous precipitation is zero except when moisture is above its saturated value. This introduces a Heaviside function that is not well represented by Fourier modes. So, when computing the precipitation spectral field, we applied a modified exponential cutoff filter using exponent $s=6, k_{\text {cut }}=10$, and $a=\log (1+400 \pi / N)$ to strongly damp small scales. In this way, the filter removes a large part of the Gibb's phenomenon. To avoid numerical instability, we had to separate the forward time stepping of the dynamics and the latent heat processes (i.e., precipitation). More precisely, the model computes the tendency term due to dynamics and advances moisture and PV. Then, it computes the precipitation field and adjusts moisture and PV accordingly to it. Finally, the divergence is computed from the PV tendencies; that is,

$$
\begin{aligned}
\boldsymbol{\nabla} \cdot \mathbf{u}_{2}= & \left(\nabla^{2}-2\right)^{-1}\left(\frac{\partial q_{1}}{\partial t}-\frac{\partial q_{2}}{\partial t}\right) \\
& +\mathbf{u}_{2} \cdot \boldsymbol{\nabla} \eta-\frac{1}{2}\left(v_{1}+v_{2}\right)-\mathcal{L} \mathcal{P} .
\end{aligned}
$$


We checked that this method gives the same result as the direct use of the omega equation, which is, in our case,

$$
\begin{aligned}
\left(-\nabla^{2}+2\right) \boldsymbol{\nabla} \cdot \mathbf{u}_{2}= & -2 \boldsymbol{\nabla} \cdot\left\{\left[\boldsymbol{\nabla}\left(\frac{\mathbf{u}_{1}+\mathbf{u}_{2}}{2}\right)\right] \boldsymbol{\nabla} \eta\right\} \\
& +\nabla^{2} \mathcal{L} \mathcal{P}+\mathcal{B}\left(v_{1}-v_{2}\right) .
\end{aligned}
$$

\section{REFERENCES}

Arbic, B. K., and G. R. Flierl, 2004: Effects of mean flow direction on energy, isotropy, and coherence of baroclinically unstable beta-plane geostrophic turbulence. J. Phys. Oceanogr., 34, 7793.

Balasubramanian, G., and M. K. Yau, 1996: The life cycle of a simulated marine cyclone: Energetics and PV diagnostics. J. Atmos. Sci., 53, 639-653.

— waves. J. Atmos. Sci., 54, 2850-2871.

Bannon, P. R., 1986: Linear development of quasi-geostrophic baroclinic disturbances with condensational heating. J. Atmos. Sci., 43, 2261-2274.

Barry, L., G. C. Craig, and J. Thuburn, 2002: Poleward heat transport by the atmospheric heat engine. Nature, 415, 774-777.

Beare, R. J., A. J. Thorpe, and A. A. White, 2003: The predictability of extratropical cyclones: Nonlinear sensitivity to localized potential-vorticity perturbations. Quart. J. Roy. Meteor. Soc., 129, 219-237.

Boer, G. J., 1995: Some dynamical consequences of greenhouse gas warming. Atmos.-Ocean, 33, 731-751.

Cao, Z., and H.-R. Cho, 1995: Generation of moist potential vorticity in extratropical cyclones. J. Atmos. Sci., 52, 3263-3281.

Danilov, S., and D. Gurarie, 2001: Forced two-dimensional turbulence in spectral and physical space. Phys. Rev. E, doi:10.1103/ PhysRevE.63.061208.

Davis, C. A., 1992: A potential vorticity diagnosis of the importance of initial structure and condensational heating in observed extratropical cyclogenesis. Mon. Wea. Rev., 120, 2409-2428.

Emanuel, K. A., 1985: Frontal circulations in the presence of small moist symmetric stability. J. Atmos. Sci., 42, 1062-1071.

— scales. J. Atmos. Sci., 53, 3276-3285.

- M. Fantini, and A. J. Thorpe, 1987: Baroclinic instability in an environment of small stability to slantwise moist convection. Part I: Two-dimensional models. J. Atmos. Sci., 44, 1559-1573.

Fantini, M., 1990: Nongeostrophic corrections to the eigensolutions of a moist baroclinic instability problem. J. Atmos. Sci., 47, $1277-1287$.

Gall, R., 1976: The effects of released latent heat in growing baroclinic waves. J. Atmos. Sci., 33, 1686-1701.

Gutowski, W. J., L. E. Branscome, and D. A. Stewart, 1992: Life cycles of moist baroclinic eddies. J. Atmos. Sci., 49, 306-319.

Gyakum, J. R., 1983: On the evolution of the QE II storm. II: Dynamics and thermodynamic structure. Mon. Wea. Rev., 111, 1156-1173.

Haidvogel, D. B., and I. M. Held, 1980: Homogeneous quasi-geostrophic turbulence driven by a uniform temperature gradient. J. Atmos. Sci., 37, 2644-2660.

Hall, N. M. J., B. J. Hoskins, P. J. Valdes, and C. A. Senior, 1994: Storm tracks in a high-resolution GCM with doubled carbon dioxide. Quart. J. Roy. Meteor. Soc., 120, 1209-1230.

Hayashi, Y., and D. G. Golder, 1981: The effects of condensational heating on midlatitude transient waves in their mature stage: Control experiments with a GFDL general circulation model. $J$. Atmos. Sci., 38, 2532-2539.
Held, I. M., 1993: Large-scale dynamics and global warming. Bull. Amer. Meteor. Soc., 74, 228-241.

_ , and V. D. Larichev, 1996: A scaling theory for horizontally homogeneous, baroclinically unstable flow on a beta plane. $J$. Atmos. Sci., 53, 946-952.

Holton, J. R., 1972: Introduction to Dynamic Meteorology. Academic Press, $319 \mathrm{pp}$.

Joly, A., and A. J. Thorpe, 1991: The stability of time-dependent flows: An application to fronts in developing baroclinic waves. J. Atmos. Sci., 48, 163-182.

Juckes, M. N., 2000: The static stability of the midlatitude troposphere: The relevance of moisture. J. Atmos. Sci., 57, 30503057.

Klein, P., A. Tréguier, and B. L. Hua, 1998: Three-dimensional stirring of thermohaline fronts. J. Mar. Res., 56, 589-612.

Lapeyre, G., and I. M. Held, 2003: Diffusivity, kinetic energy dissipation, and closure theories for the poleward eddy heat flux. J. Atmos. Sci., 60, 2907-2916.

Larichev, V. D., and I. M. Held, 1995: Eddy amplitudes and fluxes in a homogeneous model of fully developed baroclinic instability. J. Phys. Oceanogr., 25, 2285-2297.

Legg, S., and J. C. McWilliams, 2001: Convective modifications of a geostrophic eddy field. J. Phys. Oceanogr., 31, 874-891.

Mak, M., 1982: On moist quasi-geostrophic baroclinic instability. $J$. Atmos. Sci., 39, 2028-2037.

Montgomery, M. T., and B. F. Farrell, 1991: Moist surface frontogenesis associated with interior potential vorticity anomalies in a semigeostrophic model. J. Atmos. Sci., 48, 343-367.

Panetta, R. L., 1993: Zonal jets in wide baroclinically unstable regions: Persistence and scale selection. J. Atmos. Sci., 50, 2073 2106.

Pauluis, O., and I. M. Held, 2002: Entropy budget of an atmosphere in radiative-convective equilibrium. Part II: Latent heat transport and moisture processes. J. Atmos. Sci., 59, 140-149.

Pavan, V., and I. M. Held, 1996: The diffusive approximation for eddy fluxes in baroclinically unstable jets. J. Atmos. Sci., 53, $1262-1272$.

Peixoto, J. P., and A. H. Oort, 1991: Physics of Climate. American Institute of Physics, $512 \mathrm{pp}$.

Plumb, R. A., and J. D. Mahlman, 1987: The zonally averaged transport characteristics of the GFDL general circulation/transport model. J. Atmos. Sci., 44, 298-327.

Polvani, L. M., J. C. McWilliams, M. A. Spall, and R. Ford, 1994: The coherent structures of shallow-water turbulence: Deformation-radius effects, cyclone/anticyclone asymmetry and gravitywave generation. Chaos, 4, 177-186.

Rennó, N. O., and A. P. Ingersoll, 1996: Natural convection as a heat engine: A theory for CAPE. J. Atmos. Sci., 53, 572-585.

Salmon, R., 1978: Two-layer quasi-geostrophic turbulence in a simple special case. Geophys. Astrophys. Fluid Dyn., 10, 25-52.

_- 1980: Baroclinic instability and geostrophic turbulence. Geophys. Astrophys. Fluid Dyn., 15, 167-211.

Smith, K. S., G. Bocaletti, C. C. Henning, I. N. Marinov, C. Y. Tam, I. M. Held, and G. K. Vallis, 2002: Turbulent diffusion in the geostrophic inverse cascade. J. Fluid Mech., 469, 14-47.

Stephenson, D. B., and I. M. Held, 1993: GCM response of northern winter stationary waves and storm tracks to increasing amounts of carbon dioxide. J. Climate, 6, 1859-1870.

Stoelinga, M. T., 1996: A potential vorticity-based study of the role of diabatic heating and friction in a numerically simulated baroclinic cyclone. Mon. Wea. Rev., 124, 849-874.

Wang, B., and A. Barcilon, 1986: Moist stability of a baroclinic zonal flow with conditionally unstable stratification. J. Atmos. Sci., 43, 705-719.

Whitaker, J. S., and C. A. Davis, 1994: Cyclogenesis in a saturated environment. J. Atmos. Sci., 51, 889-907.

Zhang, D.-L., and R. Harvey, 1995: Enhancement of extratropical cyclogenesis by a mesoscale convective system. J. Atmos. Sci., 52, 1107-1127.

Zhang, J., 1995: Effects of latent heating on midlatitude atmospheric general circulation. Ph.D. thesis, Princeton University, 194 pp. 